\title{
A Witness for Coherent Electronic vs Vibronic-Only Oscillations in Ultrafast Spectroscopy
}

\section{Citation}

Yuen-Zhou, Joel, Jacob Krich, and Alan Aspuru-Guzik. 2012. "A witness for coherent electronic vs vibronic-only oscillations in ultrafast spectroscopy." Journal of Chemical Physics 136 (23): 234501. doi:10.1063/1.4725498. http://dx.doi.org/10.1063/1.4725498.

\section{Published Version}

doi:10.1063/1.4725498

\section{Permanent link}

http://nrs.harvard.edu/urn-3:HUL.InstRepos:11639526

\section{Terms of Use}

This article was downloaded from Harvard University's DASH repository, and is made available under the terms and conditions applicable to Other Posted Material, as set forth at http:// nrs.harvard.edu/urn-3:HUL.InstRepos:dash.current.terms-of-use\#LAA

\section{Share Your Story}

The Harvard community has made this article openly available.

Please share how this access benefits you. Submit a story.

\section{Accessibility}




\section{AIP $\begin{gathered}\text { mesoumalor } \\ \text { chemical Physics }\end{gathered}$}

A witness for coherent electronic vs vibronic-only oscillations in ultrafast spectroscopy

Joel Yuen-Zhou, Jacob J. Krich, and Alán Aspuru-Guzik

Citation: J. Chem. Phys. 136, 234501 (2012); doi: 10.1063/1.4725498

View online: http://dx.doi.org/10.1063/1.4725498

View Table of Contents: http://jcp.aip.org/resource/1/JCPSA6/v136/i23

Published by the American Institute of Physics.

Additional information on J. Chem. Phys.

Journal Homepage: http://jcp.aip.org/

Journal Information: http://jcp.aip.org/about/about_the_journal

Top downloads: http://jcp.aip.org/features/most_downloaded

Information for Authors: http://jcp.aip.org/authors

\section{ADVERTISEMENT}
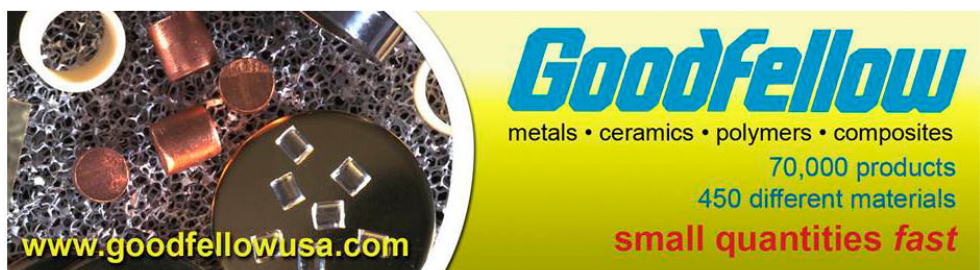


\title{
A witness for coherent electronic vs vibronic-only oscillations in ultrafast spectroscopy
}

\author{
Joel Yuen-Zhou, Jacob J. Krich, and Alán Aspuru-Guzik ${ }^{\mathrm{a})}$ \\ Department of Chemistry and Chemical Biology, Harvard University, Cambridge, Massachusetts 02138, USA
}

(Received 30 March 2012; accepted 18 May 2012; published online 18 June 2012)

\begin{abstract}
We report a conceptually straightforward witness that distinguishes coherent electronic oscillations from their vibronic-only counterparts in nonlinear optical spectra of molecular aggregates. Coherent oscillations as a function of waiting time in broadband pump/broadband probe spectra correspond to coherent electronic oscillations in the singly excited manifold. Oscillations in individual peaks of 2D electronic spectra do not necessarily yield this conclusion. Our witness is simpler to implement than quantum process tomography and potentially resolves a long-standing controversy on the character of oscillations in ultrafast spectra of photosynthetic light harvesting systems. (O 2012 American Institute of Physics. [http://dx.doi.org/10.1063/1.4725498]
\end{abstract}

\section{INTRODUCTION}

Recently, there has been considerable interest in long-lived quantum superpositions of electronic states in photosynthetic molecular aggregates and their potential role in efficient energy transport in biological conditions. ${ }^{1-7}$ Evidence for such electronic coherences stems from time oscillations in peaks of two-dimensional electronic spectra (2D-ES), which persist for over $600 \mathrm{fs}^{8-11}$ However, coherences between vibronic levels involving a single electronic state (and hence, without coherence between excited electronic states) exhibit similar signatures in 2D-ES (Refs. 12-15 and 61) and have been shown to nontrivially affect energy transfer too. ${ }^{16-25}$ Although there is additional evidence to support the interpretation that the oscillations are due to electronic states (beating frequencies and comparison with all-atom simulations ${ }^{8,26}$ ), unambiguous tools to experimentally unravel the nature of these oscillations are required. A big step has been the observation that, under weak coupling to vibrations and negligible coherence transfer processes, electronic coherences imply oscillations in off-diagonal peaks of rephasing 2D-ES and in diagonal peaks of their non-rephasing counterparts, ${ }^{27}$ whereas general vibronic coherences show up as oscillations in any region of either spectra. ${ }^{28}$ Another important proposal to address the problem in this regime has been recently suggested in Ref. 62. However, the rephasing 2D-ES of the paradigmatic Fenna-Matthews-Olson (FMO) complex exhibits oscillations in both diagonal and off-diagonal peaks, indicating that systems of interest may lie in the regime of strong coupling to vibrations ${ }^{29}$ or exhibit vibronic coherences only. ${ }^{15}$ Techniques of wavepacket reconstruction ${ }^{16,30-32}$ or quantum process tomography (QPT) (Refs. 33-35) should clearly provide an answer at a cost of several experiments. Our purpose here is to provide a practical witness for coherent electronic oscillations, which is applicable across different regimes of weak and strong coupling to vibrations.

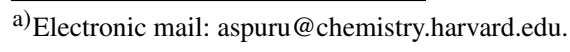

We illustrate the witness by considering the simplest molecular exciton model, the coupled dimer. ${ }^{36}$ Its Hamiltonian is given by $H_{0}(\boldsymbol{R})=T_{N}+H_{e l}(\boldsymbol{R})$, where $T_{N}$ is the nuclear kinetic energy, and $H_{e l}(\boldsymbol{R})$ is the electronic Hamiltonian which depends on the nuclei $\boldsymbol{R}, H_{e l}(\boldsymbol{R})$ $=\sum_{m n} V_{m n}(\boldsymbol{R})|m n\rangle\langle m n|+J(\boldsymbol{R})(|10\rangle\langle 01|+| 01\rangle\langle 10|) \cdot| m n\rangle$ denotes the electronic state with $m, n$ excitations in the first, second molecules, respectively $(m, n \in\{0,1\}), V_{m n}(\boldsymbol{R})$ is the corresponding diabatic potential energy surface, and $J(\boldsymbol{R})$ is the coupling between site excitations. We also introduce the convenient notation $|g\rangle=|00\rangle$ and $|f\rangle=|11\rangle$. Any pure state $|\Psi\rangle$ may be expressed in terms of vibronic states, that is, product states of the electronic (system) and nuclear (bath) degrees of freedom, $|\Psi\rangle=\sum_{i} a_{i}\left|e_{i}\right\rangle\left|N_{i}\right\rangle$, for coefficients $a_{i}$, and $\left\{\left|e_{i}\right\rangle\right\},\left\{\left|N_{i}\right\rangle\right\}$ electronic and nuclear bases. A reduced electronic description of $|\Psi\rangle$ is obtained by performing a trace over the bath, $\rho_{e l}=\operatorname{Tr}_{n u c}(|\Psi\rangle\langle\Psi|)$.

We consider a pump-probe experiment in which two non-collinear pulses interact with the sample, separated by waiting time $T$. The transmission of the probe pulse is measured, giving the pump-probe spectrum $S_{P P^{\prime}}(T)$. We treat the laser pulses as perturbations in the dipole approximation, $H_{\text {pert }}(s)=-\boldsymbol{\mu} \cdot \boldsymbol{\epsilon}(s)$, where $\boldsymbol{\mu}=\sum_{e=01,10}\left(\boldsymbol{\mu}_{e g}|e\rangle\left\langle g\left|+\boldsymbol{\mu}_{f e}\right| f\right\rangle\langle e|\right)+$ h.c. is the dipole operator, and $\boldsymbol{\epsilon}(s)=\sum_{p=P, P^{\prime}}\left[\epsilon_{p}\left(s-t_{p}\right) \boldsymbol{e}_{p}+\right.$ c.c. $]$ are the electric fields of the pump $(\mathrm{P})$ and probe $\left(\mathrm{P}^{\prime}\right)$ pulses, with $\epsilon_{p}(s)=\frac{\lambda}{\sqrt{2 \pi \sigma^{2}}} e^{-i \omega_{p} s} e^{-s^{2} / 2 \sigma^{2}}$ the Gaussian time-profile. Here, $\lambda, \omega_{p}, t_{p}, \sigma$, and $\boldsymbol{e}_{p}$, are the strength, carrier frequency, center time, width, and polarization of the $p$ th pulse, respectively. We use the Franck-Condon (FC) approximation, whereby the transition dipoles between electronic states $\left(\boldsymbol{\mu}_{e g}\right.$ and $\boldsymbol{\mu}_{f e}$ ) are constant with respect to nuclear coordinates. ${ }^{37}$ The waiting time is $T=t_{P^{\prime}}-t_{P}$, and throughout this article we set $t_{P}=0, t_{P^{\prime}}=T$. $S_{P P^{\prime}}(T)$ can also be recovered from a 2DES by integration along both frequency axes (Appendix A and Refs. 39-41).

The main result of this article is: In the FC approximation and the broadband limit $(\sigma \rightarrow 0)$, oscillations of $S_{P P^{\prime}}(T)$ as a function of $T$ correspond to coherent electronic 
oscillations. In this limit, $S_{P P^{\prime}}(T)$ may be expressed solely in terms of reduced electronic states $\rho_{e l}$ of the singly excited manifold $\{|01\rangle,|10\rangle\}$, and oscillations cannot be due exclusively to nuclear dynamics.

The $\mathrm{PP}^{\prime}$ signal can, in general, be written as

$$
S_{P P^{\prime}}(T)=S_{S E}(T)+S_{E S A}(T)+S_{G S B}(T),
$$

with separate contributions from stimulated emission (SE), excited state absorption (ESA), and ground state bleach (GSB) (Ref. 41). If the initial vibrational state is known, each of these terms may be expressed as a suitable wavefunction overlap. For example, let the initial wavefunction (before any pulse) be $\left|\Psi_{0}(0)\right\rangle=|g\rangle\left|v_{i}^{(g)}\right\rangle$, where $\left|v_{i}^{(\eta)}\right\rangle$ is a vibrational eigenstate of $H_{v i b, \eta}(\boldsymbol{R}) \equiv T_{N}+V_{\eta}(\boldsymbol{R})$. Treating the laser pulses perturbatively, the first order wavefunction due to $\mathrm{P}$ is $(\hbar=1) \quad\left|\Psi_{P}(s)\right\rangle=i \int_{-\infty}^{\infty} d s^{\prime} e^{-i H_{0}\left(s-s^{\prime}\right)}\left\{\boldsymbol{\mu} \cdot \boldsymbol{e}_{P}\left(\epsilon_{P}\left(s^{\prime}\right)\right.\right.$ + c.c. $)\}\left|\Psi_{0}\left(s^{\prime}\right)\right\rangle$, and the second order wavefunction due to both $\mathrm{P}$ and $\mathrm{P}^{\prime}$ is $\left|\Psi_{P P^{\prime}}(s)\right\rangle=i \int_{-\infty}^{\infty} d s^{\prime} e^{-i H_{0}\left(s-s^{\prime}\right)}$ $\left\{\boldsymbol{\mu} \cdot \boldsymbol{e}_{P^{\prime}}\left(\epsilon_{P^{\prime}}\left(s^{\prime}-t_{P^{\prime}}\right)+\right.\right.$ c.c. $\left.)\right\}\left|\Psi_{P}\left(s^{\prime}\right)\right\rangle$. It can be shown that each term in the signal $S_{P P^{\prime}}(T)$ may be written in terms of wavepacket overlaps (Appendix A and Refs. 37 and 42). In particular, we will focus our discussion on

$$
S_{S E}(T)=2\left\langle\Psi_{P P^{\prime}}(s) \mid g\right\rangle\left\langle g \mid \Psi_{P P^{\prime}}(s)\right\rangle,
$$

which gives the intuitive understanding that the number of photons gained by the probe $\mathrm{P}^{\prime}$ due to the action of the pump $\mathrm{P}$ is proportional to the norm of the wavepacket "dumped" into $|g\rangle$.

\section{PRELIMINARY EXAMPLE}

We will develop some intuition through an illustration, in which we focus on $S_{S E}(T)$. Consider the case where the potential energy surfaces of the singly excited diabatic states have the same shape, $V_{10}(\boldsymbol{R})=V_{01}(\boldsymbol{R})+c$, for some constant $c$ (but in general $V_{g}(\boldsymbol{R}), V_{f}(\boldsymbol{R}) \neq V_{e}(\boldsymbol{R})+c$ for $e=01,10)$, and $J(\boldsymbol{R})=J(0)$ for all $\boldsymbol{R}$. It is convenient to introduce the excitonic basis $\{|g\rangle,|\alpha\rangle,|\beta\rangle,|f\rangle\}$, which diagonalizes the electronic Hamiltonian at the ground state nuclear configuration: $H_{e l}(\mathbf{0})=\omega_{g}|g\rangle\left\langle g\left|+\omega_{\alpha}\right| \alpha\right\rangle\langle\alpha|$ $+\omega_{\beta}|\beta\rangle\left\langle\beta\left|+\omega_{f}\right| f\right\rangle\langle f|$. Here, $|g\rangle=|00\rangle$ and $|f\rangle=|11\rangle$, but in general, $|\alpha\rangle$ and $|\beta\rangle$ differ from $|01\rangle$ and $|10\rangle$ in that they are delocalized due to $J$. Note that in this example both $|\alpha\rangle$ and $|\beta\rangle$ are coupled in the same way to the vibrational bath, and hence they form a decoherence-free subspace. ${ }^{43}$ The first order wavefunction "right before" $\mathrm{P}^{\prime}$ may be expanded as $\left|\Psi_{P}(T)\right\rangle=\sum_{i=\alpha, \beta} \sum_{m} c_{i, m}(T)|i\rangle\left|v_{m}^{(i)}\right\rangle$. Since in this case, $|i\rangle\left|v_{m}^{(i)}\right\rangle$ are eigenstates of the molecular Hamiltonian $H_{0}$, the excitons are the adiabatic electronic states, there is no dissipation in the electronic system, and the values $\left|c_{i, m}(T)\right|^{2}$ are constants as a function of $T$, depending only on the details of $\mathrm{P}^{57}$ The perturbative ground-state wavepacket "right after" the probe at time $T$ is, in the FC approximation, given by

$$
\begin{aligned}
\left\langle g \mid \Psi_{P P^{\prime}}(T)\right\rangle=i & \sum_{\substack{i=\alpha, \beta \\
m n}} \boldsymbol{\mu}_{m g} \cdot \boldsymbol{e}_{P^{\prime}} \tilde{\epsilon}_{P^{\prime}}\left(\omega_{i m, g n}\right) \\
& \times\left\langle v_{n}^{(g)} \mid v_{m}^{(i)}\right\rangle c_{i, m}(T)\left|v_{n}^{(g)}\right\rangle
\end{aligned}
$$

where $\tilde{\epsilon}_{p}(\omega)=\lambda e^{-\left(\omega-\omega_{p}\right)^{2} \sigma^{2} / 2}$ is the Fourier transform of pulse $p$ at frequency $\omega, \omega_{i m, g n}$ is the energy difference between $|i\rangle\left|v_{m}^{(i)}\right\rangle$ and $|j\rangle\left|v_{n}^{(g)}\right\rangle$, and we consider only the portion of $\Psi_{P P^{\prime}}$ in the ground state; see Eq. (A5) for the full expression. This expression can be interpreted as a wavepacket in the ground state created when the probe couples the vibrational levels of the singly excited states to the vibrational levels of the ground state via the electric dipole moment, where the amplitudes in the various vibrational levels depends on the probe's electric field at the given transition energy and the FC overlap. Computing the norm of the resulting wavepacket,

$$
\begin{aligned}
S_{S E}(T)= & 2 \sum_{i j=\alpha, \beta}\left(\boldsymbol{\mu}_{i g} \cdot \boldsymbol{e}_{P^{\prime}}\right)\left(\boldsymbol{\mu}_{j g} \cdot \boldsymbol{e}_{P^{\prime}}\right) \\
& \times \sum_{m m^{\prime} n}\left\langle v_{m^{\prime}}^{(j)} \mid v_{n}^{(g)}\right\rangle\left\langle v_{n}^{(g)} \mid v_{m}^{(i)}\right\rangle \\
& \times \tilde{\epsilon}_{P^{\prime}}\left(\omega_{i m, g n}\right) \tilde{\epsilon}_{P^{\prime}}^{*}\left(\omega_{j m^{\prime}, g n}\right) c_{i, m}(T) c_{j, m^{\prime}}^{*}(T),
\end{aligned}
$$

which corresponds to sums of interferences between vibrational states of the same and different excitonic states, respectively, projecting onto the same vibrational state in the ground state.

Note that $S_{S E}(T)$ can be written as a linear combination of elements of a full vibronic density matrix $\rho(T)=\left|\Psi_{P}(T)\right\rangle\left\langle\Psi_{P}(T)\right|$. The terms $\left\langle i, m|\rho(T)| j, m^{\prime}\right\rangle$ $=c_{i m}(T) c_{j m^{\prime}}^{*}(T)$ for $(i, m) \neq\left(j, m^{\prime}\right)$ correspond to vibronic coherences and oscillate at the difference frequency between $|i\rangle|m\rangle$ and the $|j\rangle\left|m^{\prime}\right\rangle, \omega_{i m, j m^{\prime}}$. When we consider the broadband ( $b b)$ limit of Eq. (4), $\tilde{\epsilon}(\omega)=\lambda$ for all the $\omega$ values of interest, and

$$
S_{S E}^{b b}(T)=2 \lambda^{2} \sum_{\substack{i j=\alpha, \beta \\ m}}\left(\boldsymbol{\mu}_{i g} \cdot \boldsymbol{e}_{P^{\prime}}\right)\left(\boldsymbol{\mu}_{j g} \cdot \boldsymbol{e}_{P^{\prime}}\right) c_{i m}(T) c_{j m}^{*}(T)
$$

Crucially, Eq. (5) is a linear combination of elements of $\rho_{e l}(T)=\operatorname{Tr}_{n u c}\left(\left|\Psi_{P}(T)\right\rangle\left\langle\Psi_{P}(T)\right|\right.$, as opposed to the full vibronic space. The $i=j$ terms of Eq. (5) correspond to electronic populations and, due to the absence of relaxation to the ground state in this example, stay constant with respect to $T$. The term with $i=\alpha, j=\beta$ is proportional to $\left\langle\alpha\left|\rho_{e l}(T)\right| \beta\right\rangle$ $=\sum_{m} c_{\alpha, m}(T) c_{\beta, m}^{*}(T)$, so corresponds to an electronic coherence between $|\alpha\rangle$ and $|\beta\rangle$, and shows oscillations at the single frequency $\omega_{\alpha \beta}$ as a function of $T$. Hence, coherent oscillations in $S_{S E}^{b b}(T)$ are a witness for coherent electronic dynamics. Remarkably, in the additional limit where one of the excitons is dark (e.g., $\boldsymbol{\mu}_{\beta g}=0$ ), we effectively have a monomer instead of a dimer, and $S_{S E}^{b b}(T)$ is a constant even in the case of large FC displacements, where there is large vibrational motion between pump and probe. This observation for the monomer has been previously reported by Yan and Mukamel ${ }^{44}$ and recently confirmed by Mancal and coworkers. ${ }^{63}$

The results above illustrate the main point of the paper, which is summarized in Figure 1 and can be interpreted as follows. In the FC approximation, the probe couples only to the electronic dipole, so in the broadband limit it acts uniformly across every transition energy, and hence across every nuclear configuration within a particular electronic state. In general, $S_{S E}(T)$ is a sum of multiple interferences among 

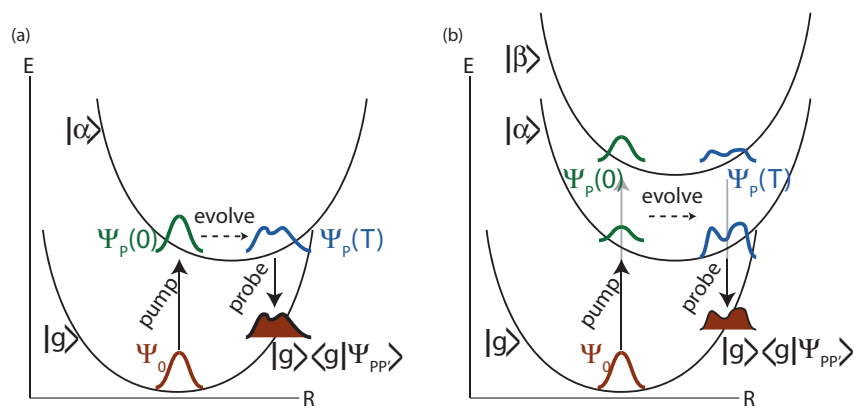

FIG. 1. Illustration of broadband pump-probe spectra as a witness for coherent electronic oscillations. (a) For a monomer with a single excited state, in the broadband $(b b)$ and Franck-Condon (FC) limits, the pump and probe pulses move amplitude in and out of the excited state regardless of the vibrational state; we observe this as leaving the shape of the vibrational wavepacket unperturbed by the pulses. Thus, the stimulated emission signal $S_{S E}^{b b}(T)$ is independent of the vibrational evolution during the waiting time $T$. (b) For a dimer with two excited states $|\alpha\rangle,|\beta\rangle$, even in the FC approximation and $b b$ limit, the two single-excitation states produce two distinct interfering quantum pathways. The signal $S_{S E}^{b b}(T)$ then oscillates at the frequency $\omega_{\alpha \beta}$ as long as electronic coherence is maintained between $|\alpha\rangle$ and $|\beta\rangle$.

portions of wavepackets at different electronic and nuclear configurations. In $S_{S E}^{b b}(T)$, the probe opens only two interference pathways (just as in the double-slit experiment), via emission from the $|\alpha\rangle$ or the $|\beta\rangle$ state, insensitive to vibrational dynamics, providing a witness for coherent electronic oscillations. The 2D-ES does not always yield this conclusion explicitly, as it includes additional information about optical vibronic coherences between the ground and excited states in the coherence and echo intervals, and therefore, is not a signal that isolates singly excited manifold dynamics.

\section{GENERAL CASE}

The example above readily generalizes to include effects of initial thermalized states of the bath, ESA and GSB contributions, and non-adiabatic effects. In the limit of broadband $P$ (Appendix C) and $\mathrm{P}^{\prime}$, the contributions of Eq. (1) to $S_{P P^{\prime}}^{b b}(T)$ are (Appendixes $\mathrm{A}$ and $\mathrm{B}$ ),

$$
\begin{aligned}
S_{S E}^{b b}(T)= & 2 \lambda^{4} \sum_{i j p q \in\{|10\rangle,|01\rangle\}}\left(\boldsymbol{\mu}_{g i} \cdot \boldsymbol{e}_{P^{\prime}}\right)\left(\boldsymbol{\mu}_{q g} \cdot \boldsymbol{e}_{P}\right) \\
& \times\left(\boldsymbol{\mu}_{g p} \cdot \boldsymbol{e}_{P}\right)\left(\boldsymbol{\mu}_{j g} \cdot \boldsymbol{e}_{P^{\prime}}\right) \chi_{i j q p}(T), \\
S_{E S A}^{b b}(T)= & -2 \lambda^{4} \sum_{i j p q \in\{|10\rangle,|01\rangle\}}\left(\boldsymbol{\mu}_{f i} \cdot \boldsymbol{e}_{P^{\prime}}\right)\left(\boldsymbol{\mu}_{q g} \cdot \boldsymbol{e}_{P}\right) \\
& \times\left(\boldsymbol{\mu}_{g p} \cdot \boldsymbol{e}_{P}\right)\left(\boldsymbol{\mu}_{j f} \cdot \boldsymbol{e}_{P^{\prime}}\right) \chi_{i j q p}(T), \\
S_{G S B}^{b b}(T)= & 2 \lambda^{4} \sum_{i p \in\{|10\rangle,|01\rangle\}}\left(\boldsymbol{\mu}_{g p} \cdot \boldsymbol{e}_{P}\right)\left(\boldsymbol{\mu}_{p g} \cdot \boldsymbol{e}_{P}\right) \\
& \times\left(\boldsymbol{\mu}_{g i} \cdot \boldsymbol{e}_{P^{\prime}}\right)\left(\boldsymbol{\mu}_{i g} \cdot \boldsymbol{e}_{P^{\prime}}\right),
\end{aligned}
$$

where the process matrix $\chi(T)$ is given by ${ }^{34,35}$

$$
\chi_{i j q p}(T)=\operatorname{Tr}_{n u c}\left\{\left\langle i\left|e^{-i H_{0} T}\left(|q\rangle\langle p| \otimes \rho_{B}(0)\right) e^{i H_{0} T}\right| j\right\rangle\right\},
$$

and it is easy to see that $S_{P P^{\prime}}^{b b}(T)$ is invariant under change of electronic basis within the singly excited states. Here, $\rho_{B}(0)=\sum_{n} p_{n}\left|v_{n}^{(g)}\right\rangle\left\langle v_{n}^{(g)}\right|$ is the initial thermal vibrational ensemble in the ground electronic state. $\chi(T)$ describes the evolution of the electronic system after $\mathrm{P}$ puts it into the singly excited manifold, and it is the central object to reconstruct in QPT (Ref. 35). If the initial state of the bath can be prepared at $\rho_{B}(0)$ regardless of the electronic state, as in the impulsive limit under consideration, an integrated equation of motion can be written for any system initial condition $\rho(0)$ as $\rho_{i j}(T)$ $=\sum_{q p} \chi_{i j q p}(T) \rho_{q p}(0)$. Note that $\chi_{i j q p}(T)$ is just a Liouville space electronic population or coherence transfer from $|i\rangle\langle j|$ (prepared by $\mathrm{P}$ ) to $|q\rangle\langle p|$ (detected by $\mathrm{P}^{\prime}$ ). As in the preliminary example, $S_{P P^{\prime}}^{b b}(T)$ is a linear combination of entries of reduced states $\rho_{e l}(T)$ of the singly excited manifold, so oscillations in it are a manifestation of electronic oscillations, justifying the witness.

Given an electronic basis, Eq. (9) indicates that any process matrix element $\chi_{i j q p}(T)$, regardless of whether it involves populations or coherences, can be oscillatory as a function of $T$, although in practice, only Fourier components that surpass a threshold in terms of amplitude and frequency will be resolved as oscillations within the timescales of the experiment. For a large variety of systems, however, it is possible to associate the largest amplitude oscillations of $\chi(T)$ to electronic coherences in some basis. In the preliminary example, the lack of dissipation implies that $\chi_{i j q p}(T)=\delta_{i q} \delta_{j p} e^{-i \omega_{q p} T}$, so the only possible oscillatory contribution to $S_{P P^{\prime}}(T)$ corresponds to $\chi_{\alpha \beta \alpha \beta}(T)=\chi_{\beta \alpha \beta \alpha}^{*}(T)$ (excitonic coherence). In the non-adiabatic case where $V_{01}(\boldsymbol{R}) \neq V_{10}(\boldsymbol{R})+c$, each electronic state couples differently to the vibrational modes. However, in the limit of weak system-bath coupling, the vibronic states $|e\rangle\left|\nu_{j}^{(e)}\right\rangle$ are still the correct eigenstates of $H_{0}$ up to zeroth order in the coupling, so any oscillations in the signal will still be dominated by excitonic coherences, if any. Finally, for intermediate and strong system-bath coupling together with a fast bath decorrelation timescale, a polaron transformation defines an electronic basis $\{|g\rangle,|\tilde{\alpha}\rangle,|\tilde{\beta}\rangle,|f\rangle\}$ that diagonalizes a zeroth-order electronic Hamiltonian weakly coupled to a renormalized bath (Ref. 45 and Appendix D). In this case, the highest amplitude oscillations in its $S_{P P^{\prime}}^{b b}(T)$ would correspond to electronic coherences $\chi_{\tilde{\alpha} \tilde{\beta} \tilde{\alpha} \tilde{\beta}}(T)=\chi_{\tilde{\beta} \tilde{\alpha} \tilde{\beta} \tilde{\alpha}}^{*}(T)$. For more general aggregates, if this were an issue of interest, a partial QPT could be designed to determine the value of specific terms of $\chi(T)$ (Refs. 34 and 35).

TABLE I. Parameters of simulations.

\begin{tabular}{cccc}
\hline \hline & $\mathrm{M}$ & $\mathrm{CD}$ & $\mathrm{ID}$ \\
\hline$E_{00} / \mathrm{cm}^{-1}$ & 0 & 0 & 0 \\
$\left\langle E_{10}-\omega_{C}\right\rangle / \mathrm{cm}^{-1}$ & -125 & -300 & -200 \\
$\left\langle E_{01}-\omega_{C}\right\rangle / \mathrm{cm}^{-1}$ & - & -200 & -200 \\
$J / \mathrm{cm}^{-1}$ & - & 100 & 10 \\
$\omega_{00, x}=\omega_{00, y} / \mathrm{cm}^{-1}$ & 100 & 100 & 100 \\
$\omega_{10, x}=\omega_{01, x} / \mathrm{cm}^{-1}$ & 200 & 200 & 200 \\
$\omega_{10, y}=\omega_{01, y} / \mathrm{cm}^{-1}$ & 150 & 150 & 150 \\
$\Delta_{00, x} / \mathrm{cm}^{1 / 2}$ & 0 & 0 & 0 \\
$\Delta_{00, y} / \mathrm{cm}^{1 / 2}$ & 0 & 0 & 0 \\
$\Delta_{10, x} / \mathrm{cm}^{1 / 2}$ & 100 & 50 & 100 \\
$\Delta_{10, y} / \mathrm{cm}^{1 / 2}$ & 0 & 0 & 0 \\
$\Delta_{01, x} / \mathrm{cm}^{1 / 2}$ & 0 & 0 & 0 \\
$\Delta_{01, y} / \mathrm{cm}^{1 / 2}$ & 0 & 50 & 100 \\
$\mathrm{FWHM}=2 \sqrt{2 \ln 2} \sigma / \mathrm{fs}$ & 10 & 18.7 & 12.5 \\
\hline \hline
\end{tabular}


(a) Monomer

(b) Coherent dimer

(c) Incoherent dimer

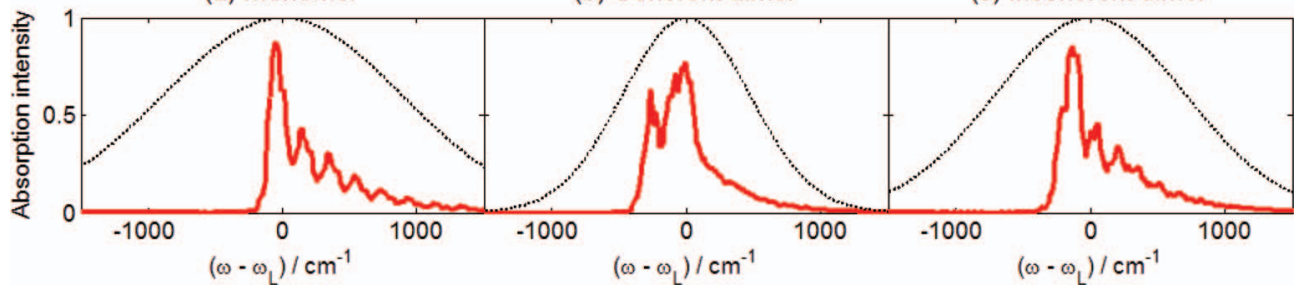

FIG. 2. Inhomogeneously broadened absorption spectra (solid red) with pulse spectral profiles $\left|\tilde{\epsilon}_{p}(\omega)\right|^{2}$ on top (dotted black).

\section{NUMERICAL EXAMPLES}

In order to illustrate the ideas in Secs. I- III, we have performed simulations for the absorption spectra, the $\mathrm{PP}^{\prime}$, and the 2D-ES for a monomer, a dimer which exhibits coherent electronic oscillations, and an incoherent dimer which does not. In all our models, each site has a single excitation and is coupled to a single vibrational mode. More explicitly, for their Hamiltonians, we choose harmonic diabatic surfaces parametrized by $V_{m n}(x, y)=E_{m n}+\frac{\omega_{m n, x}^{2}\left(x-\Delta_{m n, x}\right)^{2}}{2}+\frac{\omega_{m n, y}^{2}\left(y-\Delta_{m n, y}\right)^{2}}{2}$, where $x$ and $y$ are scaled nuclear coordinates, $E_{m n}$ are site energies, $\omega_{m n, x(y)}$ are oscillator frequencies and $\Delta_{m n, x(y)}$ are electron-nuclear couplings. ${ }^{16,17,36,42,46,47}$ We assume that the doubly excited state has no binding energy, $V_{f}(x, y)$ $=V_{10}(x, y)+V_{01}(x, y)$, and that the carrier frequency of both pulses is $\omega_{p} \equiv \omega_{C}$. The parameters for the calculations are listed in Table I. The transition dipoles of the sites are oriented $90^{\circ}$ from each other, and the ratio between their norms is 1:3. Both pulses are polarized in the same direction. All the simulations include thermal averaging of initial vibrational states according to a Boltzmann distribution at $273 \mathrm{~K}$, isotropic averaging, and explicit inclusion of pulses with the dynamics. Both the absorption spectra and the $\mathrm{PP}^{\prime}$ include inhomogeneous broadening (ensembles of 500 molecules with Gaussian site disorder of standard deviation $40 \mathrm{~cm}^{-1}$ and, for the dimers, site energy correlation 0.8 ). This latter averaging was not included for the 2D-ES due to the expensive cost of their calculation. ${ }^{58}$
Figure 2 shows absorption spectra (solid red) for the described systems as well as spectral profiles of the pulses (dotted black) used for the simulations of the witness $S_{P P^{\prime}}^{b b}(T)$ and the 2D-ES $\tilde{S}\left(\omega_{\tau}, T, \omega_{t}\right)$. These pulses approximately cover all the vibronic transitions with similar amplitude, giving a rough idea of what broadband means in practice (in these examples, a FWHM ranging from 10 to 18.7 fs). For rough estimates, there are typically two energy scales to consider, an average coupling $J$ and a reorganization energy $\lambda$, in which case the impulsive limit is set by $\frac{1}{\sigma} \gg \max (J, \lambda)$.

Figure 3 (top panel) shows $\left\langle S_{P P^{\prime}}^{b b}(T)\right\rangle$, the averaged witness as well as a few representative elements of $\chi(T)$ (bottom panel), illustrating that $S_{P P^{\prime}}^{b b}(T)$ is a direct manifestation of the dynamics of the singly excited manifold via the elements of $\chi(T)$ (see Eqs. (6)-(8)). Notice that only the coherent dimer shows oscillations of significant amplitude, yielding a positive witness. Figure 4 presents snapshots of the rephasing 2D-ES, $\left\langle\tilde{S}\left(\omega_{\tau}, T, \omega_{t}\right)\right\rangle$, for a sampling of waiting times $T$ between 71.6 and 270.6 fs (left), indicating that vibronic coherences within the same and also different excitation manifolds manifest as diagonal and cross-peak oscillations. ${ }^{59}$ Notice that due to strong coupling to vibrations, the coherent dimer also exhibits oscillations in the diagonal peaks, implying the inapplicability of previous measures for this case. ${ }^{27,28}$ As another illustration, the integrated signal under the crosspeaks encircled in black is in the right plots. Note that the largest amplitude oscillations are in the monomer, which cannot have coherent electronic oscillations for the singly excited

(a) Monomer

(b) Coherent dimer

(c) Incoherent dimer

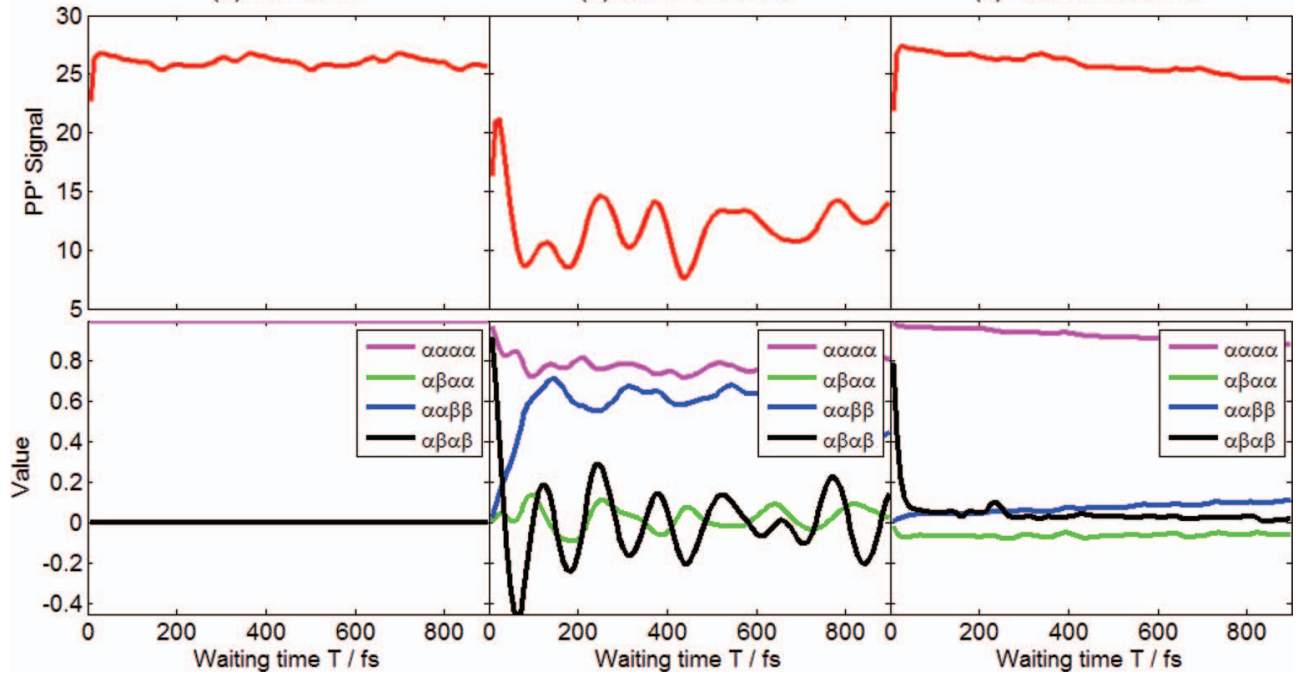

FIG. 3. (Top) Broadband $\mathrm{PP}^{\prime}$ spectra as a function of waiting time $T$ as a witness for coherent electronic oscillations. The small oscillations in (a) and (c) are due to finite pulse durations. (Bottom) The witness is a linear combination of elements of the process matrix $\chi(T)$. Traces of a few representative elements of $\chi(T)$ are displayed. 

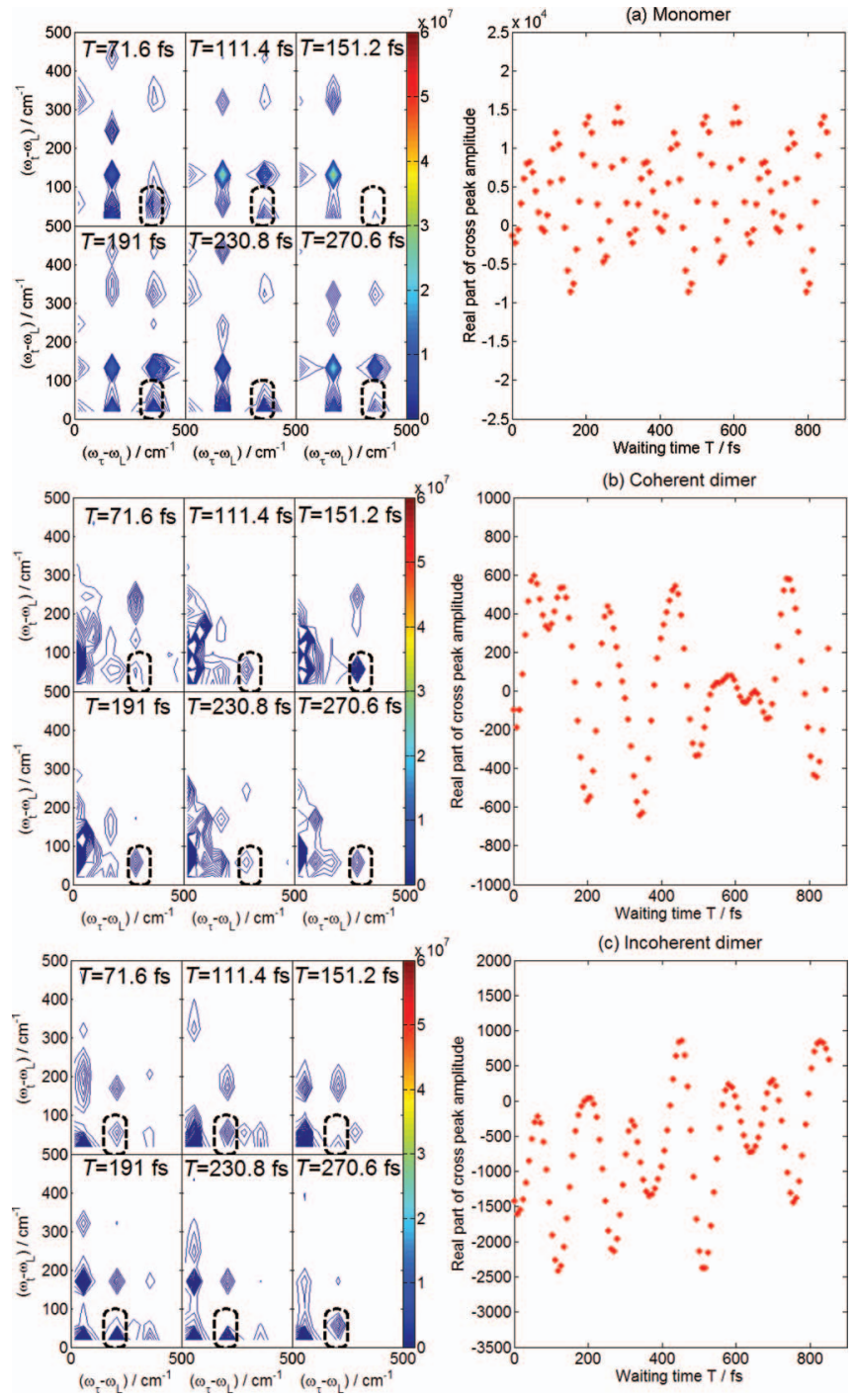

FIG. 4. (Left) Contour plot of the norm of broadband rephasing 2D-ES for (a) monomer, (b) coherent dimer, and (c) incoherent dimer. Diagonal and cross peaks oscillate as a function of time in all cases, indicating general vibronic coherences but not necessarily electronic coherence. Color scale (arbitrary units) is fixed throughout. Black dotted circles at left indicate cross peaks whose real part amplitude is shown followed in a finer time grid in the right panels (varying axes for each sample, arbitrary units). These traces are the wrong witness for coherent electronic oscillations.

manifold, showing that oscillations in peaks in the 2D-ES do not necessarily indicate coherent electronic oscillations.

Real pulses are not fully in the bb limit. The witness is positive if, once the dc background is subtracted from $S_{P P^{\prime}}^{b b}(T)$, there are oscillations with amplitude proportional to $\mu^{4}$, where $\mu$ is some estimate of an electronic transition dipole moment. If spurious oscillations due to finite pulse-duration are suspected, a more quantitative confirmation is the following: (a) Collect traces of $S_{P P}^{(\sigma)}(T)$ at several pulse widths $\sigma$, all roughly in the broadband domain. (b) Fourier transform the data: $\tilde{S}_{P P}^{(\sigma)}\left(\omega_{T}\right)=\frac{1}{2 \pi} \int_{0}^{\infty} d T e^{i \omega T} S_{P P}^{(\sigma)}(T)$. (c) Locate nonzero frequencies of $\tilde{S}_{P P}^{(\sigma)}\left(\omega_{T}\right)$ corresponding to oscillations between discrete states (ignore the dc component). For each of these frequencies $\omega_{T}$, plot $\tilde{S}_{P P}^{(\sigma)}\left(\omega_{T}\right)$ as a function of $\sigma$, and linearly extrapolate to $\sigma \rightarrow 0$. If the obtained intercepts are at zero within noise levels, the witness is negative and there are not coherent electronic oscillations. Appendix B displays an analytical expression for the $O(\sigma)$ correction of $S_{P P}^{b b}(T)$, providing a theoretical basis for this procedure. Alternatively, the ideal $S_{P P}^{b b}(T)$ can be mocked by collecting several experiments with narrowband pulses. ${ }^{48,49}$

Although the theory has been detailed here for a dimer, the witness is applicable to larger aggregates. In the case of FMO, due to spectral congestion, it might be fruitful to focus on pairs of exciton states at a time, for instance, the first and the third exciton states, ${ }^{8,10}$ either via direct $\mathrm{PP}^{\prime}$ measurements that cover these transitions exclusively, or alternatively, integrating windows of broadband 2D-ES corresponding to these two states only, assuming that relaxation processes do not occur outside of this spectral window.

In conclusion, we have provided a conceptually simple witness for coherent electronic oscillations based on broadband $\mathrm{PP}^{\prime}$ spectroscopy. We believe that this witness constitutes a timely resource which will clarify the origin of the oscillations in time-resolved spectroscopic signals of lightharvesting complexes in photosynthetic antennae, ${ }^{12-15}$ and hence provide more stringent tests on probing quantum effects in biological systems.

\section{ACKNOWLEDGMENTS}

We dedicate this paper to the late Bob Silbey who at different stages introduced J.Y.-Z. to theoretical chemistry and mentored A.A.-G. in the field. We also want to acknowledge stimulating discussions with Dylan Arias and Keith Nelson. The authors acknowledge support from the Center for Excitonics, an Energy Frontier Research Center funded by the U.S. Department of Energy, Office of Science, Office of Basic Energy Sciences under Award No. DE-SC0001088 (J.Y.-Z., J.K., A.A.-G.); Defense Advanced Research Projects Agency Grant No. N66001-10-1-4063 (A.A.-G.), Camille and Henry Dreyfus Foundation, and Alfred P. Sloan Foundation (A.A.-G.) and NSF Grant Nos. DMR-0934480 and DMR-0906475 (J.K.).

\section{APPENDIX A: PP' SIGNAL IN TERMS OF WAVEPACKET OVERLAPS}

Consider the situation described in the main text, where the total Hamiltonian is given by $H=H_{0}(\boldsymbol{R})+H_{\text {pert }}(s)$. In this section, we will assume $H_{0}(\boldsymbol{R})$ to be the same molecular piece as the one described in the main text, and $H_{\text {pert }}(s)=-\boldsymbol{\mu} \cdot \boldsymbol{\epsilon}(\boldsymbol{r}, s)$ to be the standard light-matter interaction in the dipole approximation, although we study a slightly more general setup, where the electric field is described by three (instead of just two) non-collinear beams, $\boldsymbol{\epsilon}(\boldsymbol{r}, s)=\sum_{p=1}^{3}\left[\epsilon_{p}\left(s-t_{p}\right) e^{i \boldsymbol{k}_{p} \cdot \boldsymbol{r}} \boldsymbol{e}_{p}+\right.$ c.c. $]$ where we have include the spatial modulation of the fields via the different wavevectors $\boldsymbol{k}_{p}$. The expressions for $S_{P P^{\prime}}(T)$ will appear as we take the limit of the first two pulses coinciding in time.

The pulses generate a time-dependent polarization $\boldsymbol{P}(\boldsymbol{r}, s)=\operatorname{Tr}(\boldsymbol{\mu} \rho(\boldsymbol{r}))=\sum_{\boldsymbol{k}} P(\boldsymbol{k} ; s) e^{i \boldsymbol{k} \cdot \boldsymbol{r}}$ on each molecule at position $r{ }^{60}$ The allowed wavevectors in the sum are the phase-matching directions $\boldsymbol{k}=q \boldsymbol{k}_{1}+\boldsymbol{r} \boldsymbol{k}_{2}+s \boldsymbol{k}_{3}$ for integers $q, r, s$, and encode different sequences of interactions of the pulses with the molecule. We are interested in the complex 
signal $S$ at the photon-echo (PE) phase-matched direction $\boldsymbol{k}_{P E}=-\boldsymbol{k}_{1}+\boldsymbol{k}_{2}+\boldsymbol{k}_{3}$, which can be detected by mixing the material ensemble emission with a local oscillator (LO) pulse $\boldsymbol{\epsilon}_{4}(s)$ travelling along $\boldsymbol{k}_{4}=\boldsymbol{k}_{P E}, \quad S(\tau, T, t)$ $=2 i \int_{-\infty}^{\infty} d s \epsilon_{4}^{*}\left(s-t_{4}\right) \boldsymbol{e}_{4} \cdot \boldsymbol{P}\left(\boldsymbol{k}_{P E} ; \tau, T, s\right)$, where $\tau=t_{2}-t_{1}$ (coherence time), $T=t_{3}-t_{2}$ (waiting time), and $t=t_{4}$ $-t_{3}$ (echo time) (Ref. 38). A 2D-ES $\tilde{S}\left(\omega_{\tau}, T, \omega_{t}\right)$ can be constructed as a function of $T$ by Fourier transforming the signal with respect to $\tau$ and $t, 39,41$

$$
\tilde{S}\left(\omega_{\tau}, T, \omega_{t}\right)=\int_{0}^{\infty} d \tau e^{-i \omega_{\tau} \tau} \int_{0}^{\infty} d t e^{i \omega_{t} t} S(\tau, T, t) .
$$

In general, oscillations in $S(\tau, T, t)$ and $\tilde{S}\left(\omega_{\tau}, T, \omega_{t}\right)$ can be associated to coherent superpositions of vibronic eigenstates of $H_{0}$, but not necessarily of electronic states. ${ }^{13}$ In the main text, we pay special attention to the $\mathrm{PP}^{\prime}$ limit $S_{P P^{\prime}}(T)$, which is equivalent to a differential transmission signal, where the first two pulses act as the pump P, $\left(\epsilon_{1}=\epsilon_{2}\right.$ $\left.\equiv \epsilon_{P}\right)$, the last two as the probe $\mathrm{P}^{\prime}\left(\epsilon_{3}=\epsilon_{4}=\epsilon_{P^{\prime}}\right)$, so that the coherence and echo times vanish, $\tau=t=0$, and $\mathrm{P}$ and $\mathrm{P}^{\prime}$ are well separated (i.e., $T \gg \sigma$ ). More explicitly,

$S_{P P^{\prime}}(T)=-2 \Im \int_{-\infty}^{\infty} d t^{\prime} \epsilon_{4}^{*}\left(t^{\prime}-t_{4}\right) \boldsymbol{e}_{4} \cdot \boldsymbol{P}\left(\boldsymbol{k}_{P E} ; 0, T, 0\right)$.

This limit justifies the form of $H_{\text {pert }}(s)$ given in the main text, which only consists of two pulses. Note that $S_{P P^{\prime}}(T)$ can be recovered from the 2D-ES as an inverse Fourier transform at zero frequencies, $S_{P P^{\prime}}(T)=\frac{\Re}{\pi^{2}}$ $\left\{\int_{-\infty}^{\infty} d \omega_{\tau} \int_{-\infty}^{\infty} d \omega_{t} \tilde{S}\left(\omega_{\tau}, T, \omega_{t}\right)\right\}$

We derive a wavepacket overlap formula for $S_{P P^{\prime}}(T)$ assuming that $\mathrm{P}$ and $\mathrm{P}^{\prime}$ are well separated, $T \gg \sigma$, analogously to the doorway-window approach. ${ }^{38}$ First, we conveniently define the following wavefunctions:

$$
\begin{gathered}
\left|\Psi_{0}(s)\right\rangle=e^{-i H_{0}(s)}\left|\Psi_{0}(0)\right\rangle, \\
\left|\Psi_{P}(s)\right\rangle=i \int_{-\infty}^{\infty} d s^{\prime} e^{-i H_{0}\left(s-s^{\prime}\right)}\left\{\boldsymbol{\mu} \cdot \boldsymbol{e}_{P}\left(\epsilon_{P}\left(s^{\prime}\right)+\text { c.c. }\right)\right\}\left|\Psi_{0}\left(s^{\prime}\right)\right\rangle, \\
\left|\Psi_{P P^{\prime}}(s)\right\rangle \\
=i \int_{-\infty}^{\infty} d s^{\prime} e^{-i H_{0}\left(s-s^{\prime}\right)}\left\{\boldsymbol{\mu} \cdot \boldsymbol{e}_{P^{\prime}}\left(\epsilon_{P^{\prime}}\left(s^{\prime}-T\right)+\text { c.c. }\right)\right\}\left|\Psi_{P}\left(s^{\prime}\right)\right\rangle,
\end{gathered}
$$

$$
\begin{aligned}
\left|\Psi_{P P}(s)\right\rangle= & (i)^{2} \int_{-\infty}^{\infty} d s^{\prime} \int_{-\infty}^{s^{\prime}} d s^{\prime \prime} e^{-i H_{0}\left(s-s^{\prime}\right)}\left\{\boldsymbol{\mu} \cdot \boldsymbol{e}_{P}\left(\epsilon_{P}\left(s^{\prime}\right)+\text { c.c. }\right)\right\} \\
& \times e^{-i H_{0}\left(s^{\prime}-s^{\prime \prime}\right)}\left\{\boldsymbol{\mu} \cdot \boldsymbol{e}_{P}\left(\epsilon_{P}\left(s^{\prime \prime}\right)+\text { c.c. }\right)\right\}\left|\Psi_{0}\left(s^{\prime}\right)\right\rangle,
\end{aligned}
$$

$$
\begin{aligned}
& \left|\Psi_{P^{\prime} P^{\prime}}(s)\right\rangle \\
& =(i)^{2} \int_{-\infty}^{\infty} d s^{\prime} \int_{-\infty}^{s^{\prime}} d s^{\prime \prime} e^{-i H_{0}\left(s-s^{\prime}\right)}\left\{\boldsymbol{\mu} \cdot \boldsymbol{e}_{P^{\prime}}\left(\epsilon_{P^{\prime}}\left(s^{\prime}-T\right)+\text { c.c. }\right)\right\} \\
& \times e^{-i H_{0}\left(s^{\prime}-s^{\prime \prime}\right)}\left\{\boldsymbol{\mu} \cdot \boldsymbol{e}_{P^{\prime}}\left(\epsilon_{P^{\prime}}\left(s^{\prime \prime}-T\right)+\text { c.c. }\right)\right\}\left|\Psi_{0}\left(s^{\prime \prime}\right)\right\rangle, \quad \text { (A7) } \\
& \left|\Psi_{P P P^{\prime}}(s)\right\rangle=(i) \int_{-\infty}^{\infty} d s^{\prime} e^{-i H_{0}\left(s-s^{\prime}\right)}\left\{\boldsymbol { \mu } \cdot \boldsymbol { e } _ { P ^ { \prime } } \left(\epsilon_{P^{\prime}}\left(s^{\prime}-T\right)\right.\right. \\
& + \text { c.c. })\}\left|\Psi_{P P}\left(s^{\prime}\right)\right\rangle,
\end{aligned}
$$

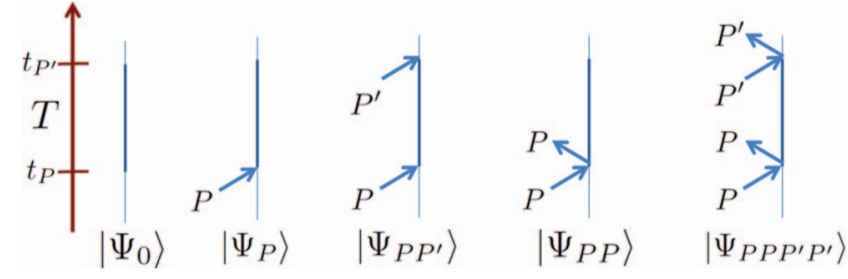

FIG. 5. Feynman diagrams for the wavefunctions defined in Eqs. (A3)-(A9).

$$
\begin{aligned}
\left|\Psi_{P P P^{\prime} P^{\prime}}(s)\right\rangle= & (i) \int_{-\infty}^{\infty} d s^{\prime} e^{-i H_{0}\left(s-s^{\prime}\right)}\left\{\boldsymbol { \mu } \cdot \boldsymbol { e } _ { P ^ { \prime } } \left(\epsilon_{P^{\prime}}\left(s^{\prime}-T\right)\right.\right. \\
& + \text { c.c. })\}\left|\Psi_{P P P^{\prime}}\left(s^{\prime}\right)\right\rangle,
\end{aligned}
$$

which are valid for $s \gg T$ (after the envelope of the last pulse has substantially decayed), and which correspond to the processes indicated by their subscripts, i.e., $\left|\Psi_{P P P^{\prime} P^{\prime}}(s)\right\rangle$ corresponds to the fourth order wavefunction $\left(O\left(\lambda^{4}\right)\right)$ resulting from two actions of $\mathrm{P}$ and two of $\mathrm{P}^{\prime}$ (see Fig. 5).

Equations (A3)-(A9) allow for a calculation of $\boldsymbol{P}\left(\boldsymbol{k}_{P E} ; 0, T, 0\right)$ and hence of $S_{P P^{\prime}}(T)$ via Eq. (A2). The phase-matching condition $\boldsymbol{k}_{P E}=-\boldsymbol{k}_{1}+\boldsymbol{k}_{2}+\boldsymbol{k}_{3}$ together with the rotating-wave approximation indicate that for each wavevector $+\boldsymbol{k}_{j}\left(-\boldsymbol{k}_{j}\right)$, the pulse $j$ acts with the term $\epsilon_{j}\left(\epsilon_{j}^{*}\right)$, exciting (de-exciting) the ket or de-exciting (exciting) the bra. Collecting all the terms gives $S_{P P^{\prime}}(T)=S_{S E}(T)$ $+S_{E S A}(T)+S_{G S B}(T)$ :

$$
\begin{aligned}
S_{S E}(T) & \\
= & -2 \Im \int_{-\infty}^{\infty} d t^{\prime}\left\langle\Psi_{P P^{\prime}}\left(t^{\prime}\right)\left|\left\{\epsilon_{P^{\prime}}^{*}\left(t^{\prime}-T\right) \boldsymbol{\mu} \cdot \boldsymbol{e}_{P^{\prime}}\right\}\right| \Psi_{P}\left(t^{\prime}\right)\right\rangle \\
= & -2 \Im(-i) \int_{-\infty}^{\infty} d t^{\prime} \int_{-\infty}^{t^{\prime}} d s^{\prime}\left\langle\Psi_{P}\left(s^{\prime}\right)\right|\left\{-i \epsilon_{P^{\prime}}\left(s^{\prime}-T\right) \boldsymbol{\mu} \cdot \boldsymbol{e}_{P^{\prime}}\right\} \\
& \times e^{i H_{0}\left(t^{\prime}-s^{\prime}\right)}\left\{(i) \epsilon_{P^{\prime}}^{*}\left(t^{\prime}-T\right) \boldsymbol{\mu} \cdot \boldsymbol{e}_{P^{\prime}}\right\}\left|\Psi_{P}\left(t^{\prime}\right)\right\rangle \\
= & 2\left\langle\Psi_{P P^{\prime}}(s) \mid g\right\rangle\left\langle g \mid \Psi_{P P^{\prime}}(s)\right\rangle,
\end{aligned}
$$

\section{$S_{E S A}(T)$}

$$
\begin{aligned}
= & -2 \Im \int_{-\infty}^{\infty} d t^{\prime}\left\langle\Psi_{P}\left(t^{\prime}\right)\left|\left\{\epsilon_{P^{\prime}}^{*}\left(t^{\prime}-T\right) \boldsymbol{\mu} \cdot \boldsymbol{e}_{P^{\prime}}\right\}\right| \Psi_{P P^{\prime}}\left(t^{\prime}\right)\right\rangle \\
= & -2 \Im(i) \int_{-\infty}^{\infty} d t^{\prime} \int_{-\infty}^{t^{\prime}} d s^{\prime}\left\langle\Psi_{P}\left(t^{\prime}\right)\right|\left\{(-i) \epsilon_{P^{\prime}}^{*}\left(t^{\prime}-T\right) \boldsymbol{\mu} \cdot \boldsymbol{e}_{P^{\prime}}\right\} \\
& \times e^{-i H_{0}\left(t^{\prime}-s^{\prime}\right)}\left\{i \epsilon_{P^{\prime}}\left(s^{\prime}-T\right) \boldsymbol{\mu} \cdot \boldsymbol{e}_{P^{\prime}}\right\}\left|\Psi_{P}\left(s^{\prime}\right)\right\rangle \\
= & -2\left\langle\Psi_{P P^{\prime}}(s) \mid f\right\rangle\left\langle f \mid \Psi_{P P^{\prime}}(s)\right\rangle,
\end{aligned}
$$

$S_{G S B}(T)$

$$
\begin{aligned}
= & -2 \Im \int_{-\infty}^{\infty} d t^{\prime}\left\{\left\langle\Psi_{P P}\left(t^{\prime}\right)\left|\left\{\epsilon_{P^{\prime}}^{*}\left(t^{\prime}-T\right) \boldsymbol{\mu} \cdot \boldsymbol{e}_{P^{\prime}}\right\}\right| \Psi_{P^{\prime}}\left(t^{\prime}\right)\right\rangle\right. \\
& \left.+\left\langle\Psi_{0}\left(t^{\prime}\right)\left|\left\{\epsilon_{P^{\prime}}^{*}\left(t^{\prime}-T\right) \boldsymbol{\mu} \cdot \boldsymbol{e}_{P^{\prime}}\right\}\right| \Psi_{P P P^{\prime}}\left(t^{\prime}\right)\right\rangle\right\} \\
= & -2 \Im(-i) \int_{-\infty}^{\infty} d t^{\prime}\left\{\left\langle\Psi_{P P}\left(t^{\prime}\right)\left|\left\{(i) \epsilon_{P^{\prime}}^{*}\left(t^{\prime}-T\right) \boldsymbol{\mu} \cdot \boldsymbol{e}_{P^{\prime}}\right\}\right| \Psi_{P^{\prime}}\left(t^{\prime}\right)\right\rangle\right. \\
& \left.+\left\langle\Psi_{0}\left(t^{\prime}\right)\left|\left\{(i) \epsilon_{P^{\prime}}^{*}\left(t^{\prime}-T\right) \boldsymbol{\mu} \cdot \boldsymbol{e}_{P^{\prime}}\right\}\right| \Psi_{P P P^{\prime}}\left(t^{\prime}\right)\right\rangle\right\} \\
= & 2 \Re\left\{\left\langle\Psi_{P P}(s) \mid g\right\rangle\left\langle g \mid \Psi_{P^{\prime} P^{\prime}}(s)\right\rangle+\left\langle\Psi_{0}(s) \mid g\right\rangle\left\langle g \mid \Psi_{P P P^{\prime} P^{\prime}}(s)\right\rangle\right\},
\end{aligned}
$$


where again, $s \gg T$, and otherwise, the signals are independent of $s$. This can be understood in physical terms: after the action of the pulses, the wavefunctions still evolve according to $H_{0}$, but the overlaps do not change in time. Equations (A10)-(A12) are in the spirit of wavepacket approaches to $\mathrm{PP}^{\prime}$ spectroscopy. ${ }^{16-18,42,50-52}$

In order to gain additional insight, we interpret the formulas in terms of differential transmission by enumerating all the possible absorption and emission processes which are quadratic in $\mathrm{P}$ and $\mathrm{P}^{\prime}$. $\mathrm{P}$ promotes a wavepacket from $|g\rangle$ to $\left|\Psi_{P}(s)\right\rangle$, a superposition of wavepackets in $|\alpha\rangle$ and $|\beta\rangle . \mathrm{P}^{\prime}$ acts on this state, creating $\left|\Psi_{P P^{\prime}}(s)\right\rangle$, a superposition of wavepackets in $|g\rangle$ and $|f\rangle$. Naturally, the photons emitted in SE correspond to the squared amplitude of $\left\langle g \mid \Psi_{P P^{\prime}}(t)\right\rangle$, whereas the ones absorbed in ESA are associated with the squared amplitude of $\left\langle f \mid \Psi_{P P^{\prime}}(s)\right\rangle$, hence providing an intuition for the expressions for $S_{S E}(T)$ and $S_{E S A}(T)$. Finally, $S_{G S B}(T)$ can be thought as accounting for the "leftover" SE processes, namely, overlaps between wavepackets created by pulses at different times. After $\mathrm{P}$ and $\mathrm{P}^{\prime}$, the total ground state wavepacket is $\langle g \mid \Psi(t)\rangle=\left\langle g\left|\left(\left|\Psi_{0}(t)\right\rangle+\left|\Psi_{P P^{\prime}}(t)\right\rangle+\left|\Psi_{P P}(t)\right\rangle+\left|\Psi_{P^{\prime} P^{\prime}}(t)\right\rangle\right.\right.\right.$ $+\left|\Psi_{P P P^{\prime} P^{\prime}}(t)\right\rangle+$ higher order contributions). Collecting wavepacket overlaps which are quadratic in both pulses yields $S_{S E}(T)+S_{G S B}(T)$. "Leftover" ESA processes do not contribute to $S_{P P^{\prime}}(T)$ because they do not fulfill the PE phase-matching condition (they appear in double-quantum coherence spectroscopy, for instance).

Thermal effects follow from averaging the signals corresponding to initial states $\left|\Psi_{0}(0)\right\rangle$ sampled according to a Boltzmann distribution.

\section{APPENDIX B: GENERAL EXPRESSIONS FOR $S_{P P^{\prime}}(T)$ IN VIBRONIC BASIS}

In order to manipulate the wavepacket overlap expressions in Appendix A, it is convenient to define two vibronic bases:
- The vibronic eigenbasis of $H_{0},\left\{\left|g, v_{n}^{(g)}\right\rangle,|\zeta\rangle,\left|f, v_{n}^{(f)}\right\rangle\right\}$, which satisfy

$$
\begin{aligned}
H_{0}\left|g, v_{n}^{(g)}\right\rangle & =\omega_{g n}\left|g, v_{n}^{(g)}\right\rangle, \\
H_{0}|\zeta\rangle & =\omega_{\zeta}|\zeta\rangle, \\
H_{0}\left|f, v_{n}^{(f)}\right\rangle & =\omega_{f n}\left|f, v_{n}^{(f)}\right\rangle,
\end{aligned}
$$

where $|\zeta\rangle$ is any of the vibronic states of the singly excited manifold.

- The tensor product basis $\left\{\left|m, v_{n}^{(g)}\right\rangle\right\}$, where $\{|m\rangle\}$ denotes electronic states in an arbitrary electronic basis (for instance, the excitonic one), and $\left\{\left|v_{n}^{(g)}\right\rangle\right\}$ refers to vibrational eigenstates of the ground vibrational Hamiltonian, $H_{v i b, 00}(\boldsymbol{R})=T_{N}+V_{00}(\boldsymbol{R})$.

It is always possible to write states in the vibronic eigenbasis in terms of the second one: $\left|g, v_{n}^{(g)}\right\rangle$ stays the same, $|\zeta\rangle=\sum_{m n}\left\langle m, v_{n}^{(g)} \mid \zeta\right\rangle\left|m, v_{n}^{(g)}\right\rangle$, and $\left|f, v_{n^{\prime}}^{(f)}\right\rangle$ $=\sum_{n}\left|f, v_{n^{\prime}}^{(g)}\right\rangle\left\langle f, v_{n}^{(g)} \mid f, v_{n^{\prime}}^{(f)}\right\rangle=\sum_{n}\left\langle v_{n}^{(g)} \mid v_{n^{\prime}}^{(f)}\right\rangle\left|f, v_{n^{\prime}}^{(g)}\right\rangle$. Using both bases, the process matrix (see Eq. (9)) affords a compact representation,

$$
\begin{aligned}
\chi_{i j q p}(T)= & \operatorname{Tr}_{n u c}\left\{\left\langle i\left|e^{-i H_{0} T}\left(|q\rangle\langle p| \otimes \rho_{B}(0)\right) e^{i H_{0} T}\right| j\right\rangle\right\} \\
= & \sum_{\zeta \zeta^{\prime} n n^{\prime}} p_{n} e^{-i\left(\omega_{\zeta}-\omega_{\zeta^{\prime}}\right) T}\left\langle i, v_{n^{\prime}}^{(g)} \mid \zeta\right\rangle\left\langle\zeta \mid q, v_{n}^{(g)}\right\rangle \\
& \times\left\langle p, v_{n}^{(g)} \mid \zeta^{\prime}\right\rangle\left\langle\zeta^{\prime} \mid j, v_{n^{\prime}}^{(g)}\right\rangle
\end{aligned}
$$

Our goal is to express $S_{P P^{\prime}}(T)$ for arbitrary bandwidth in a similar style, so that in the broadband limit, we can identify it as a linear combinations of elements of $\chi(T)$, hence proving Eqs. (6)-(8). We start by rewriting Eqs. (A3)-(A9) in the vibronic bases:

$$
\begin{aligned}
\left|\Psi_{P P^{\prime}}(s)\right\rangle= & -\sum_{i q}\left(\boldsymbol{\mu}_{g i} \cdot \boldsymbol{e}_{P^{\prime}}\right)\left(\boldsymbol{\mu}_{q g} \cdot \boldsymbol{e}_{P}\right) \sum_{\zeta n^{\prime}}\left\langle i, v_{n^{\prime}}^{(g)} \mid \zeta\right\rangle\left\langle\zeta \mid q, v_{n}^{(g)}\right\rangle e^{-i \omega_{\zeta}\left(s-t_{1}\right)} \\
& \times\left(\sum_{m}\left\langle v_{m}^{(f)}\left|v_{n^{\prime}}^{(g)}\right| \tilde{\epsilon}_{P^{\prime}}\left(\omega_{f m, \zeta}\right) \tilde{\epsilon}_{P}\left(\omega_{\zeta, g n}\right) \mid f\right\rangle+\tilde{\epsilon}_{P^{\prime}}\left(\omega_{\zeta, g n^{\prime}}\right) \tilde{\epsilon}_{P}\left(\omega_{\zeta, g n}\right)|g\rangle\right)\left|v_{n}^{(g)}\right\rangle, \\
\left|\Psi_{P P}(s)\right\rangle= & -\sum_{i q}\left(\boldsymbol{\mu}_{i g} \cdot \boldsymbol{e}_{P}\right)\left(\boldsymbol{\mu}_{q g} \cdot \boldsymbol{e}_{P}\right) \sum_{\zeta n^{\prime}}\left\langle i, v_{n^{\prime}}^{(g)} \mid \zeta\right\rangle\left\langle\zeta\left|q, v_{n}^{(g)}\right| e^{-i \omega_{g n^{\prime}}\left(s-t_{1}\right)}\right. \\
& \times \frac{1}{2} \tilde{\epsilon}_{P}\left(\omega_{g n^{\prime}, \zeta}\right) \tilde{\epsilon}_{P}\left(\omega_{\zeta, g n}\right)\left(1-\operatorname{Erf}\left(\frac{i \sigma\left(\left(\omega_{C}+\omega_{g n^{\prime}, \zeta}\right)+\left(\omega_{C}-\omega_{\zeta, g n}\right)\right)}{2}\right)\right)|g\rangle\left|v_{n^{\prime}}^{(g)}\right\rangle, \\
\left|\Psi_{P^{\prime} P^{\prime}}(s)\right\rangle= & -\sum_{j p}\left(\boldsymbol{\mu}_{i g} \cdot \boldsymbol{e}_{P^{\prime}}\right)\left(\boldsymbol{\mu}_{p g} \cdot \boldsymbol{e}_{P^{\prime}}\right) \sum_{\zeta n^{\prime}}\left\langle j, v_{n^{\prime}}^{(g)} \mid \zeta\right\rangle\left\langle\zeta \mid p, v_{n}^{(g)}\right\rangle e^{-i \omega_{g n^{\prime}}\left(s-t_{2}\right)} \\
& \times \frac{1}{2} \tilde{\epsilon}_{P}\left(\omega_{g n^{\prime}, \zeta}\right) \tilde{\epsilon}_{P}\left(\omega_{\zeta, g n}\right)\left(1-\operatorname{Erf}\left(\frac{i \sigma\left(\left(\omega_{C}+\omega_{g n^{\prime}, \zeta}\right)+\left(\omega_{C}-\omega_{\zeta, g n}\right)\right)}{2}\right)\right)|g\rangle\left|v_{n^{\prime}}^{(g)}\right\rangle,
\end{aligned}
$$




$$
\begin{aligned}
\left|\Psi_{P P P^{\prime} P^{\prime}}(s)\right\rangle= & \sum_{i j q p}\left(\boldsymbol{\mu}_{g j} \cdot \boldsymbol{e}_{P^{\prime}}\right)\left(\boldsymbol{\mu}_{p g} \cdot \boldsymbol{e}_{P^{\prime}}\right)\left(\boldsymbol{\mu}_{g i} \cdot \boldsymbol{e}_{P}\right)\left(\boldsymbol{\mu}_{q g} \cdot \boldsymbol{e}_{P}\right) \\
& \times \sum_{\zeta^{\prime} \zeta n n^{\prime} n^{\prime \prime}}\left\langle j, v_{n^{\prime \prime}}^{(g)} \mid \zeta^{\prime}\right\rangle\left\langle\zeta^{\prime} \mid p, v_{n^{\prime}}^{(g)}\right\rangle\left\langle i, v_{n^{\prime}}^{(g)} \mid \zeta\right\rangle\left\langle\zeta \mid q, v_{n}^{(g)}\right\rangle e^{-i \omega_{g n^{\prime}}\left(s-t_{1}\right)} \\
& \times \frac{1}{4} \tilde{\epsilon}_{P^{\prime}}\left(\omega_{g n^{\prime \prime}, \zeta^{\prime}}\right) \tilde{\epsilon}_{P^{\prime}}\left(\omega_{\zeta^{\prime}, g n^{\prime}}\right) \tilde{\epsilon}_{P}\left(\omega_{g n^{\prime}, \zeta}\right) \tilde{\epsilon}_{P}\left(\omega_{\zeta, g n}\right) \\
& \times\left(1-\operatorname{Erf}\left(\frac{i \sigma\left(\left(\omega_{C}+\omega_{g n^{\prime \prime}, \zeta}\right)+\left(\omega_{C}-\omega_{\zeta^{\prime}, g n^{\prime}}\right)\right.}{2}\right)\right) \\
& \times\left(1-\operatorname{Erf}\left(\frac{i \sigma\left(\left(\omega_{C}+\omega_{g n^{\prime}, \zeta}\right)+\left(\omega_{C}-\omega_{\zeta, g n}\right)\right.}{2}\right)\right)|g\rangle\left|v_{n^{\prime \prime}}^{(g)}\right\rangle,
\end{aligned}
$$

where the Erf functions appear due to pulse overlap. Equations (A10)-(A12) together with Eqs. (B5)-(B8) yield

$$
\begin{aligned}
& S_{S E}(T)=\sum_{i j q p}\left(\boldsymbol{\mu}_{g i} \cdot \boldsymbol{e}_{P^{\prime}}\right)\left(\boldsymbol{\mu}_{q g} \cdot \boldsymbol{e}_{P}\right)\left(\boldsymbol{\mu}_{g p} \cdot \boldsymbol{e}_{P}\right)\left(\boldsymbol{\mu}_{j g} \cdot \boldsymbol{e}_{P^{\prime}}\right) \sum_{\zeta \zeta^{\prime} n n^{\prime}} \tilde{\epsilon}_{P^{\prime}}\left(\omega_{g n^{\prime}, \zeta}\right) \tilde{\epsilon}_{P}\left(\omega_{\zeta, g n}\right) \tilde{\epsilon}_{P}\left(\omega_{g n, \zeta^{\prime}}\right) \tilde{\epsilon}_{P^{\prime}}\left(\omega_{\zeta^{\prime}, g n^{\prime}}\right) \\
& \times p_{n} e^{-i\left(\omega_{\zeta}-\omega_{\zeta^{\prime}}\right) T}\left\langle i, v_{n^{\prime}}^{(g)} \mid \zeta\right\rangle\left\langle\zeta \mid q, v_{n}^{(g)}\right\rangle\left\langle p, v_{n}^{(g)} \mid \zeta^{\prime}\right\rangle\left\langle\zeta^{\prime} \mid j, v_{n^{\prime}}^{(g)}\right\rangle \\
& S_{E S A}(T)=-\sum_{i j q p}\left(\boldsymbol{\mu}_{f i} \cdot \boldsymbol{e}_{P^{\prime}}\right)\left(\boldsymbol{\mu}_{q g} \cdot \boldsymbol{e}_{P}\right)\left(\boldsymbol{\mu}_{g p} \cdot \boldsymbol{e}_{P}\right)\left(\boldsymbol{\mu}_{j f} \cdot \boldsymbol{e}_{P^{\prime}}\right) \\
& \times \sum_{\zeta \zeta^{\prime} n n^{\prime} n^{\prime \prime} m}\left\langle v_{n^{\prime \prime}}^{(g)} \mid v_{m}^{(f)}\right\rangle\left\langle v_{m}^{(f)}\left|v_{n^{\prime}}^{(g)}\right| \tilde{\epsilon}_{P^{\prime}}\left(\omega_{f m, \zeta}\right) \tilde{\epsilon}_{P}\left(\omega_{\zeta, g n}\right) \tilde{\epsilon}_{P}\left(\omega_{g n, \zeta^{\prime}}\right) \tilde{\epsilon}_{P^{\prime}}\left(\omega_{\zeta^{\prime}, f m}\right)\right. \\
& \times p_{n} e^{-i\left(\omega_{\zeta}-\omega_{\zeta^{\prime}}\right) T}\left\langle i, v_{n^{\prime}}^{(g)} \mid \zeta\right\rangle\left\langle\zeta \mid q, v_{n}^{(g)}\right\rangle\left\langle p, v_{n}^{(g)} \mid \zeta^{\prime}\right\rangle\left\langle\zeta^{\prime} \mid j, v_{n^{\prime \prime}}^{(g)}\right\rangle, \\
& S_{G S B}(T)=2 \Re \sum_{i j q p}\left(\boldsymbol{\mu}_{g i} \cdot \boldsymbol{e}_{P^{\prime}}\right)\left(\boldsymbol{\mu}_{q g} \cdot \boldsymbol{e}_{P^{\prime}}\right)\left(\boldsymbol{\mu}_{g p} \cdot \boldsymbol{e}_{P}\right)\left(\boldsymbol{\mu}_{j g} \cdot \boldsymbol{e}_{P}\right)\left(\frac{1}{4}\right) \sum_{\zeta \zeta n n^{\prime}} \tilde{\epsilon}_{P^{\prime}}\left(\omega_{g n^{\prime}, \zeta}\right) \tilde{\epsilon}_{P^{\prime}}\left(\omega_{\zeta, g n}\right) \tilde{\epsilon}_{P}\left(\omega_{g n, \zeta^{\prime}}\right) \tilde{\epsilon}_{P}\left(\omega_{\zeta^{\prime}, g n^{\prime}}\right) \\
& \times\left\{p_{n}\left\langle i, v_{n^{\prime}}^{(g)} \mid \zeta\right\rangle\left\langle\zeta \mid q, v_{n}^{(g)}\right\rangle\left\langle p, v_{n}^{(g)} \mid \zeta^{\prime}\right\rangle\left\langle\zeta^{\prime} \mid j, v_{n^{\prime}}^{(g)}\right\rangle e^{-i \omega_{g n, g n^{\prime}} T}\right. \\
& \left.\times\left(1-\operatorname{Erf}\left(\frac{i \sigma\left(\left(-\omega_{g n^{\prime}, \zeta}-\omega_{C}\right)+\left(\omega_{\zeta, g n}-\omega_{C}\right)\right)}{2}\right)\right)\left(1-\operatorname{Erf}\left(\frac{i \sigma\left(\left(-\omega_{g n, \zeta^{\prime}}-\omega_{C}\right)+\left(\omega_{\zeta^{\prime}, g n^{\prime}}-\omega_{C}\right)\right)}{2}\right)\right)^{*}\right) \\
& +p_{n}\left\langle i, v_{n^{\prime}}^{(g)} \mid \zeta\right\rangle\left\langle\zeta \mid q, v_{n}^{(g)}\right\rangle\left(\left\langle p, v_{n}^{(g)} \mid \zeta^{\prime}\right\rangle\left\langle\zeta^{\prime}\left|j, v_{n^{\prime}}^{(g)}\right| e^{i \omega_{g n^{\prime}, g n} T}\right)^{*}\right. \\
& \left.\times\left(1-\operatorname{Erf}\left(\frac{i \sigma\left(\left(-\omega_{g n^{\prime}, \zeta}-\omega_{C}\right)+\left(\omega_{\zeta, g n}-\omega_{C}\right)\right)}{2}\right)\right)\left(1-\operatorname{Erf}\left(\frac{i \sigma\left(\left(-\omega_{g n, \zeta^{\prime}}-\omega_{C}\right)+\left(\omega_{\zeta^{\prime}, g n^{\prime}}-\omega_{C}\right)\right)}{2}\right)\right)\right\}
\end{aligned}
$$

The expressions above can be intuitively understood in terms of the double-sided Feynman diagrams in Fig. 6. The expression for GSB consists of a sum of terms corresponding to two types of Feynman pathways, which are different in general.

In the broadband limit where $\tilde{\epsilon}_{P}(\omega)=\tilde{\epsilon}_{P^{\prime}}(\omega)=\lambda$, many sums above collapse through resolutions of the identity, and we straightforwardly recover Eqs. (6)-(8). In this limit, as highlighted by the $T$-independent form of Eq. (8), the two types of GSB pathways yield the same stationary background to the signal (caused by copies of the initial stationary wavepackets in the ground electronic surface).

In the practical case where the pulses are broad, but not infinitely sharp in time, the leading (first) order correction in $\sigma$ comes from the pulse-overlaps (Erf-functions) in the GSB 
term. We can expand, $S_{P P^{\prime}}(T)=S_{P P^{\prime}}^{b b}(T)+S_{G S B}^{(1)}(T)$,

$$
\begin{aligned}
S_{G S B}^{(1)}(T)= & -\frac{\sigma \lambda^{4}}{2} \sum_{i j q p}\left(\boldsymbol{\mu}_{g i} \cdot \boldsymbol{e}_{P^{\prime}}\right)\left(\boldsymbol{\mu}_{q g} \cdot \boldsymbol{e}_{P^{\prime}}\right)\left(\boldsymbol{\mu}_{g p} \cdot \boldsymbol{e}_{P}\right)\left(\boldsymbol{\mu}_{j g} \cdot \boldsymbol{e}_{P}\right) \\
& \times \Re\left\{\sum_{\zeta \zeta n n^{\prime}} p_{n}\left\langle i, v_{n^{\prime}}^{(g)} \mid \zeta\right\rangle\left\langle\zeta \mid q, v_{n}^{(g)}\right\rangle\left\langle p, v_{n}^{(g)} \mid \zeta^{\prime}\right\rangle\left\langle\zeta^{\prime} \mid j, v_{n^{\prime}}^{(g)}\right\rangle e^{-i \omega_{g n, g n^{\prime}} T}\right. \\
& \times \frac{i\left(\left(-\omega_{g n^{\prime}, \zeta}-\omega_{C}\right)+\left(\omega_{\zeta, g n}-\omega_{C}\right)\right)}{\sqrt{\pi}}+\frac{-i\left(\left(-\omega_{g n, \zeta^{\prime}}-\omega_{C}\right)+\left(\omega_{\zeta^{\prime}, g n^{\prime}}-\omega_{C}\right)\right)}{\sqrt{\pi}} \\
& +\sum_{\zeta \zeta n n^{\prime}} p_{n}\left\langle i, v_{n^{\prime}}^{(g)} \mid \zeta\right\rangle\left\langle\zeta \mid q, v_{n}^{(g)}\right\rangle\left(\left\langle p, v_{n}^{(g)} \mid \zeta^{\prime}\right\rangle\left\langle\zeta^{\prime} \mid j, v_{n^{\prime}}^{(g)}\right\rangle e^{i \omega_{g n^{\prime}, g n} T}\right)^{*} \\
& \left.+\frac{i\left(\left(-\omega_{g n^{\prime}, \zeta}-\omega_{C}\right)+\left(\omega_{\zeta, g n}-\omega_{C}\right)\right)}{\sqrt{\pi}}+\frac{i\left(\left(-\omega_{g n, \zeta^{\prime}}-\omega_{C}\right)+\left(\omega_{\zeta^{\prime}, g n^{\prime}}-\omega_{C}\right)\right)}{\sqrt{\pi}}\right\} .
\end{aligned}
$$

SE and ESA processes only contribute to corrections of $O\left(\sigma^{2}\right)$ via the Gaussian spectral profile of the pulses.

\section{APPENDIX C: REQUIREMENT OF BROADBAND PUMP P}

Although the conclusions of the preliminary example in the main text hold even in the case of narrowband $\mathrm{P}$, we also require broad bandwidth for $\mathrm{P}$ for two reasons:

1. Non-stationary GSB contributions. Equations (A6) and (A9) show that in the limit of broadband $\mathrm{P}$, this pulse promotes a wavepacket to the excited states and immediately back down to $|g\rangle$, yielding a wavefunction $\left|\Psi_{P P}(s)\right\rangle$ that is proportional to the original $\left|\Psi_{0}(0)\right\rangle$ before any pulse (also see Eq. (8)). In this limit, as emphasized in Appendix A, $S_{G S B}(T)$ yields a constant background as a function of $T$, giving the opportunity to identify $S_{P P^{\prime}}(T)$ as a selective probe for singly excited state dynamics, as opposed to a measurement which also includes information about dynamics in $|g\rangle$ or $|f\rangle$. Under a narrowband $\mathrm{P}$, this no longer holds, as shown by Eq. (B11), which depends on $T$ in general. In this

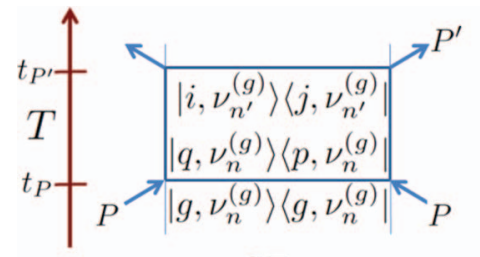

$S E$

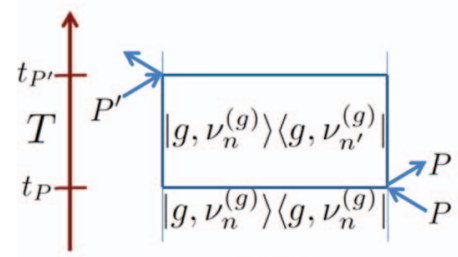

$G S B$

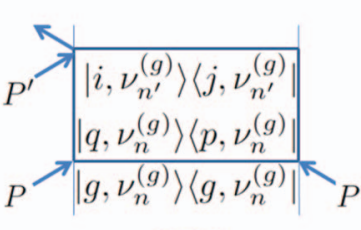

ESA

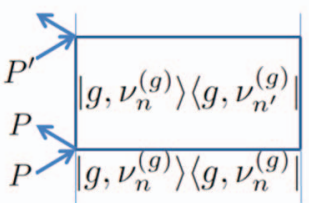

GSB
FIG. 6. Double-sided Feynman diagrams for general PP' signal. case, $\left|\Psi_{P P}(s)\right\rangle$ will be a non-stationary wavepacket in the ground electronic surface, which will manifest as time-evolving overlaps both in $\left\langle\Psi_{P P}(s) \mid g\right\rangle\left\langle g \mid \Psi_{P^{\prime} P^{\prime}}(s)\right\rangle$ and in $\left\langle\Psi_{0}(s) \mid g\right\rangle\left\langle g \mid \Psi_{P P P^{\prime} P^{\prime}}(s)\right\rangle$ (see Eq. (A12)).

2. Consistency with $Q P T$. As mentioned in the main text, the initial states prepared under a broadband $\mathrm{P}$ are of the form $\rho(0)=|q\rangle\langle p| \otimes \rho_{B}(0)$, where $\rho_{B}(0)$ $=\sum_{n} p_{n}\left|v_{n}^{(g)}\right\rangle\left\langle v_{n}^{(g)}\right|$ is the initial thermal ensemble of vibrations in the ground electronic surface for all $|q\rangle\langle p|$. Under a narrowband $\mathrm{P}$, it is not possible to prepare initial tensor product states between the system and a fixed bath $\rho_{B}$, so $S_{P P^{\prime}}(T)$ can no longer be written in terms of elements of a single $\chi(T)$, and the equation $\rho(T)=\chi(T) \rho(0)$ loses its meaning.

\section{APPENDIX D: POLARON TRANSFORMATION}

Here, we summarize the essential features of the polaron transformation used in the arguments of the article. We closely follow the works of Silbey, Harris, and coworkers. ${ }^{45,53,54}$ Consider the approximation where the diabatic potential energy surfaces are given by harmonic wells along each nuclear coordinate, $V_{10}(\boldsymbol{R})=E_{10}+\sum_{n} \frac{m_{n} \omega_{n}^{2} R_{n}^{2}}{2}$ $+\sqrt{2 m_{n} \omega_{n}^{3}} g_{10, n} R_{n}$, and $V_{01}(\boldsymbol{R})$ has the same form except for the substitution $10 \rightarrow 01$ in the subscripts, whereas $V_{g}(\boldsymbol{R})$ and $V_{f}(\boldsymbol{R})$ have arbitrary shapes. Here, $\omega_{n}$ denotes the $n$th mode frequency, whereas the displacements $g_{10, n}$ denote linear couplings of the electronic system to the nuclear bath. Define the harmonic oscillator creation and anhi-

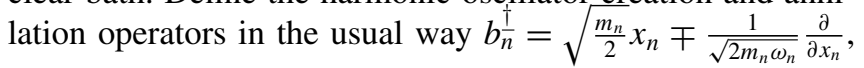
and also the generator $G=\sum_{n}\left(b_{n}^{\dagger}-b_{n}\right)\left(g_{10, n}|10\rangle\langle 10|\right.$ $\left.+g_{01, n}|01\rangle\langle 01|\right)$ such that $U=e^{G}$ corresponds to a unitary transformation of the full-polaron transformation. ${ }^{54}$ It follows that $\tilde{H}(\boldsymbol{R}) \equiv e^{G} H_{0}(\boldsymbol{R}) e^{-G}=\tilde{H}_{0}(\boldsymbol{R})+\tilde{H}_{1}(\boldsymbol{R})$, where $\tilde{H}_{0}(\boldsymbol{R})=T_{N}+\tilde{H}_{e l}(\boldsymbol{R})$ is our new zeroth-order Hamiltonian, and $\tilde{H}_{1}(\boldsymbol{R})$ is the perturbation term, whenever it is small compared to $\tilde{H}_{0}(\boldsymbol{R})$. To make a connection with the previous notation, we explicitly write $\tilde{H}_{e l}=\sum_{m n} \tilde{V}_{m n}|m n\rangle\langle m n|$ 
$+\tilde{J}(|10\rangle\langle 01|+| 01\rangle\langle 10|)$, where,

$$
\begin{aligned}
\tilde{V}_{10} & =E_{10}-\sum_{n} \omega_{n} g_{10, n}^{2}+\sum_{n} \frac{m_{n} \omega_{n}^{2} R_{n}^{2}}{2}, \\
\tilde{V}_{01} & =E_{01}-\sum_{n} \omega_{n} g_{01, n}^{2}+\sum_{n} \frac{m_{n} \omega_{n}^{2} R_{n}^{2}}{2}, \\
\tilde{J} & =J\langle w\rangle, \\
w & =\exp \left(\sum_{n}\left(g_{10, n}-g_{01, n}\right)\left(b_{n}^{\dagger}-b_{n}\right)\right), \\
\langle w\rangle & =\operatorname{Tr}\left(w \rho_{B}(0)\right) \\
& =\exp \left(-\sum_{n} \operatorname{coth} \frac{\beta \omega_{n}\left(g_{n D}-g_{n A}\right)^{2}}{2}\right) .
\end{aligned}
$$

The expressions $\beta, \rho_{B}(0)=\prod_{n} \sum_{r} \frac{\exp \left(-\beta \omega_{n}\left(r+\frac{1}{2}\right)\right)\left|\omega_{n}, r\right\rangle\left\langle\omega_{n}, r\right|}{Z_{n}(\beta)}$, $Z_{n}(\beta)=\frac{1}{2 \sinh \left(\beta \omega_{n} / 2\right)},\left|\omega_{n}, r\right\rangle$ label the inverse temperature, the initial thermal ensemble of vibrations, the partition function of the $n$th oscillator, and the $r$ th eigenstate of the $n$th harmonic oscillator, respectively. $\tilde{J}$ can be interpreted as a renormalized site-site coupling due to phonon-dressing. Furthermore, $\tilde{H}_{1}(\boldsymbol{R})=J(w-\langle w\rangle)|10\rangle\langle 01|+$ h.c. Jang advises to consider the smallness of the quantity $J \sqrt{1-w^{2}}$ as the figure of merit for the validity of perturbation theory, and hence for the usefulness of the polaron transformation. ${ }^{54} \mathrm{Cao}$ and co-workers note that the accuracy of the polaron transformation is guaranteed only in the scenario of fast bath decorrelation compared to the other relevant timescales. ${ }^{55}$

If the dynamics of all the degrees of freedom are governed by $\tilde{H}_{0}(\boldsymbol{R})$ alone, the electronic system is effectively uncoupled from the nuclear bath. The diagonalization of $\tilde{H}_{0}(0)$ yields polaronic states $\{|g\rangle,|\tilde{\alpha}\rangle,|\tilde{\beta}\rangle,|f\rangle\}$ which satisfy $\chi_{i j q p}(T)=\delta_{i q} \delta_{j p} e^{-i \omega_{q p} T}$. As can be easily checked, Eqs. (6)(8) are invariant under change of basis. Hence, if $\tilde{H}_{1}(\boldsymbol{R})$ can be guaranteed to be a small perturbation for $\tilde{H}_{0}(\boldsymbol{R})$, to zerothorder in $\tilde{H}_{1}(\boldsymbol{R})$, the coherent electronic oscillations in $S_{P P^{\prime}}^{b b}(T)$ correspond to electronic coherences in the polaronic basis.

The steps above have been outlined for the full-polaron transformation, but the conclusion can be easily seen to hold whenever the total Hamiltonian can be repartitioned into a large contribution and a small system-bath coupling. Examples include the variational polaron transformation, ${ }^{45,53}$ which interpolates between weak and strong coupling between the original system and bath, as well transformations which include anharmonicities in the diabatic potential energy surfaces (quadratic coupling between the original system and bath $\left.^{56}\right)$.

\footnotetext{
${ }^{1}$ M. Mohseni, P. Rebentrost, S. Lloyd, and A. Aspuru-Guzik, J. Chem. Phys. 129, 174106 (2008).

${ }^{2}$ M. B. Plenio and S. F. Huelga, New J. Phys. 10, 113019 (2008).

${ }^{3}$ J. Cao and R. J. Silbey, J. Phys. Chem. A 113, 13825-13838 (2009).

${ }^{4}$ P. Huo and D. F. Coker, J. Phys. Chem. Lett. 2(7), 825-833 (2011).

${ }^{5}$ L. A. Pachón and P. Brumer, J. Phys. Chem. Lett. 2(21), 2728-2732 (2011).

${ }^{6}$ M. Sarovar, A. Ishizaki, G. R. Fleming, and K. B. Whaley, Nat. Phys. 6, 462 (2010).

${ }^{7}$ C. Kreisbeck and T. Kramer, e-print arXiv:1203.1485.
}

${ }^{8}$ G. S. Engel, T. R. Calhoun, E. L. Read, T. K. Ahn, T. Mancal, Y. C. Cheng, R. E. Blankenship, and G. R. Fleming, Nature (London) 446, 782-786 (2007)

${ }^{9}$ E. Collini, C. Y. Wong, K. E. Wilk, P. M. G. Curmi, P. Brumer, and G. D. Scholes, Nature (London) 463, 644-U69 (2010).

${ }^{10}$ G. Panitchayangkoon, D. Hayes, K. A. Fransted, J. R. Caram, E. Harel, J. Wen, R. E. Blankenship, and G. S. Engel, Proc. Natl. Acad. Sci. U.S.A. 107(29), 12766-12770 (2010).

${ }^{11}$ D. Hayes, J. Wen, G. Panitchayangkoon, R. E. Blankenship, and G. S. Engel, Faraday Discuss. 150, 459-469 (2011).

${ }^{12}$ D. Egorova, M. F. Gelin, and W. Domcke, J. Chem. Phys. 126, 074314 (2007).

${ }^{13}$ D. Egorova, Chem. Phys. 347(1-3), 166-176 (2008).

${ }^{14}$ N. Christensson, F. Milota, J. Hauer, J. Sperling, O. Bixner, A. Nemeth, and H. F. Kauffmann, J. Phys. Chem. B 115(18), 5383-5391 (2011).

${ }^{15} \mathrm{~N}$. Christensson, H. F. Kauffmann, T. Pullerits, and T. Mancal, e-print arXiv:1201.6325.

${ }^{16}$ J. D. Biggs and J. A. Cina, J. Chem. Phys. 131, 224101 (2009).

${ }^{17}$ J. D. Biggs and J. A. Cina, J. Chem. Phys. 131, 224302 (2009).

${ }^{18}$ J. D. Biggs and J. A. Cina, J. Phys. Chem. A 116(7), 1683-1693 (2012).

${ }^{19}$ F. Caycedo-Soler, A. W. Chin, J. Almeida, S. F. Huelga, and M. B. Plenio, J. Chem. Phys. 136, 155102 (2012).

${ }^{20}$ A. W. Chin, J. Prior, R. Rosenbach, F. Caycedo-Soler, S. F. Huelga, and M. B. Plenio, e-print arXiv:1203.0776.

${ }^{21}$ J. M. Womick and A. M. Moran, J. Phys. Chem. B 115(6), 1347-1356 (2011).

${ }^{22}$ S. Polyutov, O. Kuhn, and T. Pullerits, Chem. Phys. 394(1), 21-28 (2012).

${ }^{23}$ A. Kolli, E. J. O'Reilly, G. D. Scholes, and A. Olaya-Castro, e-print arXiv: 1203.5056.

${ }^{24}$ E. Collini and G. D. Scholes, J. Phys. Chem. A 113(16), 4223-4241 (2009).

${ }^{25}$ M. F. Gelin, L. Z. Sharp, D. Egorova, and W. Domcke, J. Chem. Phys. 136(3), 034507 (2012)

${ }^{26} \mathrm{~S}$. Shim, P. Rebentrost, S. Valleau, and A. Aspuru-Guzik, Biophys. J. 102(3), 649-660 (2012).

${ }^{27}$ Y. C. Cheng and G. R. Fleming, J. Phys. Chem. A 112, 4254-4260 (2008).

${ }^{28}$ D. B. Turner, K. E. Wilk, P. M. G. Curmi, and G. D. Scholes, J. Phys. Chem. Lett. 2(15), 1904-1911 (2011).

${ }^{29}$ G. Panitchayangkoon, D. V. Voronine, D. Abramavicius, J. R. Caram, N. H. C. Lewis, S. Mukamel, and G. S. Engel, Proc. Natl. Acad. Sci. U.S.A. 108(52), 20908-20912 (2011).

${ }^{30}$ T. S. Humble and J. A. Cina, Phys. Rev. Lett. 93, 060402 (2004).

${ }^{31}$ D. Avisar and D. Tannor, Phys. Rev. Lett. 106, 170405 (2011).

${ }^{32}$ D. Avisar and D. Tannor, Faraday Discuss. 153, 131 (2011).

${ }^{33}$ P. Rebentrost, S. Shim, J. Yuen-Zhou, and A. Aspuru-Guzik, Procedia Chem. 3(1), 332 (2011).

${ }^{34}$ J. Yuen-Zhou and A. Aspuru-Guzik, J. Chem. Phys. 134(13), 134505 (2011).

${ }^{35}$ J. Yuen-Zhou, J. J. Krich, M. Mohseni, and A. Aspuru-Guzik, Proc. Natl. Acad. Sci. U.S.A. 108(43), 17615 (2011).

${ }^{36}$ T. Forster, Delocalized Excitation and Energy Transfer (Academic, New York, 1965), Vol. 3, pp. 93-137.

${ }^{37}$ D. J. Tannor, Introduction to Quantum Mechanics: A Time Dependent Approach (University Science Books, 2007).

${ }^{38}$ S. Mukamel, Principles of Nonlinear Optical Spectroscopy (Oxford University Press, 1995).

${ }^{39}$ S. Mukamel, Ann. Rev. Phys. Chem. 51(1), 691-729 (2000).

${ }^{40}$ M. Cho, Chem. Rev. 108, 1331-1418 (2008).

${ }^{41}$ M. Cho, Two Dimensional Optical Spectroscopy (CRC, 2009).

${ }^{42}$ J. A. Cina and G. R. Fleming, J. Phys. Chem. A 108(51), 11196-11208 (2004).

${ }^{43}$ D. A. Lidar, I. L. Chuang, and K. B. Whaley, Phys. Rev. Lett. 81, 25942597 (1998).

${ }^{44}$ Y. J. Yan and S. Mukamel, Phys. Rev. A 41, 6485-6504 (1990).

${ }^{45}$ R. Silbey and R. A. Harris, J. Chem. Phys. 80(6), 2615-2617 (1984).

${ }^{46}$ A. Eisfeld, L. Braun, W. T. Strunz, J. S. Briggs, J. Beck, and V. Engel, J. Chem. Phys. 122(13), 134103 (2005).

${ }^{47}$ J. A. Cina, D. S. Kilin, and T. S. Humble, J. Chem. Phys. 118, 46-61 (2003).

${ }^{48}$ N. E. Henriksen and V. Engel, J. Chem. Phys. 111(23), 10469-10475 (1999).

${ }^{49}$ N. E. Henriksen and V. Engel, Int. Rev. Phys. Chem. 20(2), 93-126 (2001).

${ }^{50} \mathrm{~S}$. Mukamel, C. Ciordas-Ciurdariu, and V. Khidekel, Time-Frequency and Coordinate-Momentum Wigner Wavepackets in Nonlinear Spectroscopy (Wiley, 2007), pp. 345-372. 
${ }^{51}$ J. Cao and K. R. Wilson, "Detecting wave packet motion in pump-probe," J. Chem. Phys. 106(12), 5062-5072 (1997).

${ }^{52}$ S. Y. Lee, Wave-Packet Model of Dynamic Dispersed and Integrated PumpProbe Signals in Femtosecond Transition-State Spectroscopy (Wiley-VCH Verlag $\mathrm{GmbH}, 2008$ ), pp. 273-298.

${ }^{53}$ R. A. Harris and R. Silbey, J. Chem. Phys. 83(3), 1069-1074 (1985).

${ }^{54}$ S. Jang, J. Chem. Phys. 131(16), 164101 (2009).

${ }^{55}$ C. Kong Lee, J. Moix, and J. Cao, "Accuracy of second order perturbation theory in the polaron and variational polaron frames," e-print arXiv:1201.2436.

${ }^{56}$ R. W. Munn and R. Silbey, J. Chem. Phys. 68(5), 2439-2450 (1978).

${ }^{57}$ This is within the assumptions of the model; e.g., $T$ must be shorter than the fluorescence timescale of approximately $1 \mathrm{~ns}$.
${ }^{58}$ Details of the computational methodology will be presented elsewhere.

${ }^{59}$ Inhomogeneous broadening was not included in the 2D-ES due to the expensive cost of their computation.

${ }^{60} \mathrm{We}$ use the word polarization in two different ways: To denote (a) the orientation of oscillations of the electric field and (b) the density of electric dipole moments in a material. The meaning should be clear by the context.

${ }^{61}$ V. Butkus, L. Valkunas, and D. Abramavicius, "Molecular vibrationsinduced quantum beats in two-dimensional electronic spectroscopy," e-print arXiv:1205.3383.

${ }^{62}$ V. Butkus, D. Zigmantas, L. Valkunas, and D. Abramavicius, "Vibrational vs. electronic coherences in 2D spectrum of molecular systems," e-print arXiv:1201.2753.

${ }^{63}$ T. Mancal, N. Christensson, V. Lukes, F. Milota, O. Bixner, H. Kauffmann, and J. Hauer, J. Phys. Chem. Lett. 3, 1497 (2012). 